\title{
Friction-Induced Nanofabrication: A Review
}

\author{
Bingjun Yu and Linmao Qian*
}

\begin{abstract}
As the bridge between basic principles and applications of nanotechnology, nanofabrication methods play significant role in supporting the development of nanoscale science and engineering, which is changing and improving the production and lifestyle of the human. Photo lithography and other alternative technologies, such as nanoimprinting, electron beam lithography, focused ion beam cutting, and scanning probe lithography, have brought great progress of semiconductor industry, IC manufacturing and micro/nanoelectromechanical system (MEMS/NEMS) devices. However, there remains a lot of challenges, relating to the resolution, cost, speed, and so on, in realizing high-quality products with further development of nanotechnology. None of the existing techniques can satisfy all the needs in nanoscience and nanotechnology at the same time, and it is essential to explore new nanofabrication methods. As a newly developed scanning probe microscope (SPM)-based lithography, friction-induced nanofabrication provides opportunities for maskless, flexible, low-damage, low-cost and environment-friendly processing on a wide variety of materials, including silicon, quartz, glass surfaces, and so on. It has been proved that this fabrication route provides with a broad application prospect in the fabrication of nanoimprint templates, microfluidic devices, and micro/nano optical structures. This paper hereby involved the principals and operations of friction-induced nanofabrication, including friction-induced selective etching, and the applications were reviewed as well for looking ahead at opportunities and challenges with nanotechnology development. The present review will not only enrich the knowledge in nanotribology, but also plays a positive role in promoting SPM-based nanofabrication.
\end{abstract}

Keywords: Scanning probe microscope, Tip-based lithography, Friction-induced nanofabrication, Friction-induced selective etching

\section{Introduction}

Nanoscience and nanotechnology have brought significant improvement of our live quality in wide field from electronic gadgets to healthcare and medical devices. As an important part in triggering the development of nanotechnology, nanofabrication plays a key role in realizing micro/nano-scale structures and device with novel properties in optical, electronic, magnetic or other behaviors. Not only micro- and nanolithography has been the main driving technology in semiconductor and integrated circuit (IC) industry, it also plays an increasingly important role in manufacturing of commercial microelectromechanical system (MEMS) devices as well as prototype

\footnotetext{
*Correspondence: linmao@swjtu.edu.cn

Tribology Research Institute, State Key Laboratory of Traction Power, Southwest Jiaotong University, Chengdu 610031, China
}

fabrication in emerging nanoscale science and engineering [1]. Depending on whether the template is used or not, nanolithography techniques are divided into two types, i.e., masked and maskless lithography. The masked lithography is suitable for large-area manufacturing by transferring patterns from templates or molds, and includes photolithography [2], soft lithography [3], and nanoimprint [4]. In contrast, maskless lithography can be more flexible in fabricating arbitrary patterns by directly writing or scanning without any mask, including electron beam lithography (EBL) [5], focused ion beam lithography (FIBL) $[6,7]$, and scanning probe lithography (SPL) $[8,9]$. However, none of the existing techniques can satisfy all the needs at the same time with the nanotechnology developing towards diversification and depth [10].

With high resolution and flexibility, scanning probe lithography has attracted great attention worldwide. In
Springer Open

(c) The Author(s) 2021. This article is licensed under a Creative Commons Attribution 4.0 International License, which permits use, sharing, adaptation, distribution and reproduction in any medium or format, as long as you give appropriate credit to the original author(s) and the source, provide a link to the Creative Commons licence, and indicate if changes were made. The images or other third party material in this article are included in the article's Creative Commons licence, unless indicated otherwise in a credit line to the material. If material is not included in the article's Creative Commons licence and your intended use is not permitted by statutory regulation or exceeds the permitted use, you will need to obtain permission directly from the copyright holder. To view a copy of this licence, visit http://creativeco mmons.org/licenses/by/4.0/. 
1982, Dr. Binnig of IBM Zurich Research Laboratory and his colleagues jointly invented the world's first scanning tunneling microscope (STM) [11]. This invention makes it possible for human beings to observe atoms in the atmosphere and at room temperature for the first time. STM and subsequent atomic force microscopy (AFM) have become powerful tools for nanotechnology research, and promoting an important enabling technology, i.e., scanning probe-based nanolithography (SPL) or tip-based nanofabrication (TBN). SPL can perform wide types of manufacturing activities, from material removal and modification to material deposition and manipulation, all in the nanoscale [12].

Some pioneering works were realized by scanning probe microscopy (SPM), including STM and AFM. According to different processing principles, SPM lithography mainly involves single atom manipulation, mechanical removal, local anodizing, dip-pen printing, thermal probe machining, etc $[12,13]$. With a SPM tip, surface atoms could be manipulated controllably via adjusting the tunneling current or interfacial force between the tip and the sample [14-18]. Tip-induced local anodic oxidation (LAO) process can be performed under a given bias voltage, and the contact area of a conductive surface is oxidized, forming oxide patterns corresponding to the designed tip traces $[19,20]$. Dip-pen nanolithography (DPN), a nanofabrication method proposed by Mirkin et al. in the 1990s, can transfer "ink" molecules to substrate surface to write specific nanostructures using AFM tip as a pen, and has promising applications in the fields of physics, chemistry, biology and medical treatment $[21,22]$. A SPL method based on the local desorption of a glassy organic resist by a heatable probe was proposed for fabricating nonpatterns at a half pitch down to 15 $\mathrm{nm}$ without proximity corrections, and complex threedimensional structures can be duplicated from a photograph [23]. SPL was also used for fabricating micro/ nanostructures on a flat surface by mechanical removal, but the tip can suffer wear and become blunt. Despite much progress in AFM nanolithography, there are still a lot of problems related to the resolution, speed, cost, smear, and so on, yet to be solved [10].

In the past decade, friction-induced nanofabrication was proposed to produce nanostructures on surfaces of wide types of materials, including silicon, gallium arsenide, glass, quartz, and so on $[24,25]$. For the formation of hillocks by directly tip scratching, the friction and shear are the necessary conditions for the generation of hillocks [26]. Furthermore, the friction-induced hillock and groove-shaped scratches created under low-normalload scratching can act as a mask resisting against or promoting the etching, i.e., friction-induced selective etching [27]. Considering that the friction and shear contribute greatly to the final formation of low-damage nanostructures either by direct scratching or by post selective etching, the processes of friction-induced hillock and friction-induced selective etching can be hereby viewed as friction-induced nanofabrication. Figure 1 presents the development of friction-induced nanofabrication with some representative events. Compared to the traditional nanofabrication methods such as photolithography and nanoimprinting, friction-induced nanofabrication has excellent properties of low cost, flexibility, and low damage. During the tip scanning, the trace for tip scanning can be programmed in terms of a demanded pattern, and hence it has no challenges in producing different patterns at specified locations without templates [24]. Large-scale and high-resolution fabrication can be realized through multi-probe scanning technology.

This paper will review the principals and operations of friction-induced nanofabrication, including frictioninduced selective etching. Aside from direct nanofabrication with AFM tip scratching, mechanical or tribochemical mechanisms of material deformation or damages stemming from tip scratching, and the roles of different structures or phases in selective etching were address for fully intercepting the low-damage structure formation. Some examples for the applications of the fabrication were presented as well for looking ahead at opportunities and challenges for this nanofabrication method.

\section{Direct Nanofabrication with AFM Tip Scratching}

\subsection{Direct Nanofabrication by Mechanical Removal}

Mechanical SPL technique presents high resolution and low cost for directly fabricating various surface nanostructures (e.g., nanodots, nanogrooves, and 2D/3D nanostructures) through surface material removal. The lithography is provided with two modes, i.e., static and dynamic plowing lithography (DPL), which were respectively operated with contact and tapping modes. With static plowing lithography, so-called scratching, various nanostructures can be realized on sample surfaces by applying a preset normal load on an AFM tip. For instance, using a closed-loop nanoscale precision stage integrated with an AFM, 3D nanostructures with complex geometry (e.g., 3D human face shape, nanoline arrays of sine-wave and triangular profiles, nanodot arrays of sine-shaped, hemispheric, and concave/convex nanopatterns) were successfully realized on aluminum surface according to predetermined designs in a controllable and reproducible fashion (Figure 2a) [28]. These nanostructures can hardly be fabricated by bottom-up processes at low cost and with high accuracy. Another excellent example was the rapid fabrication of complex 3D nanostructures on poly (methyl methacrylate) 


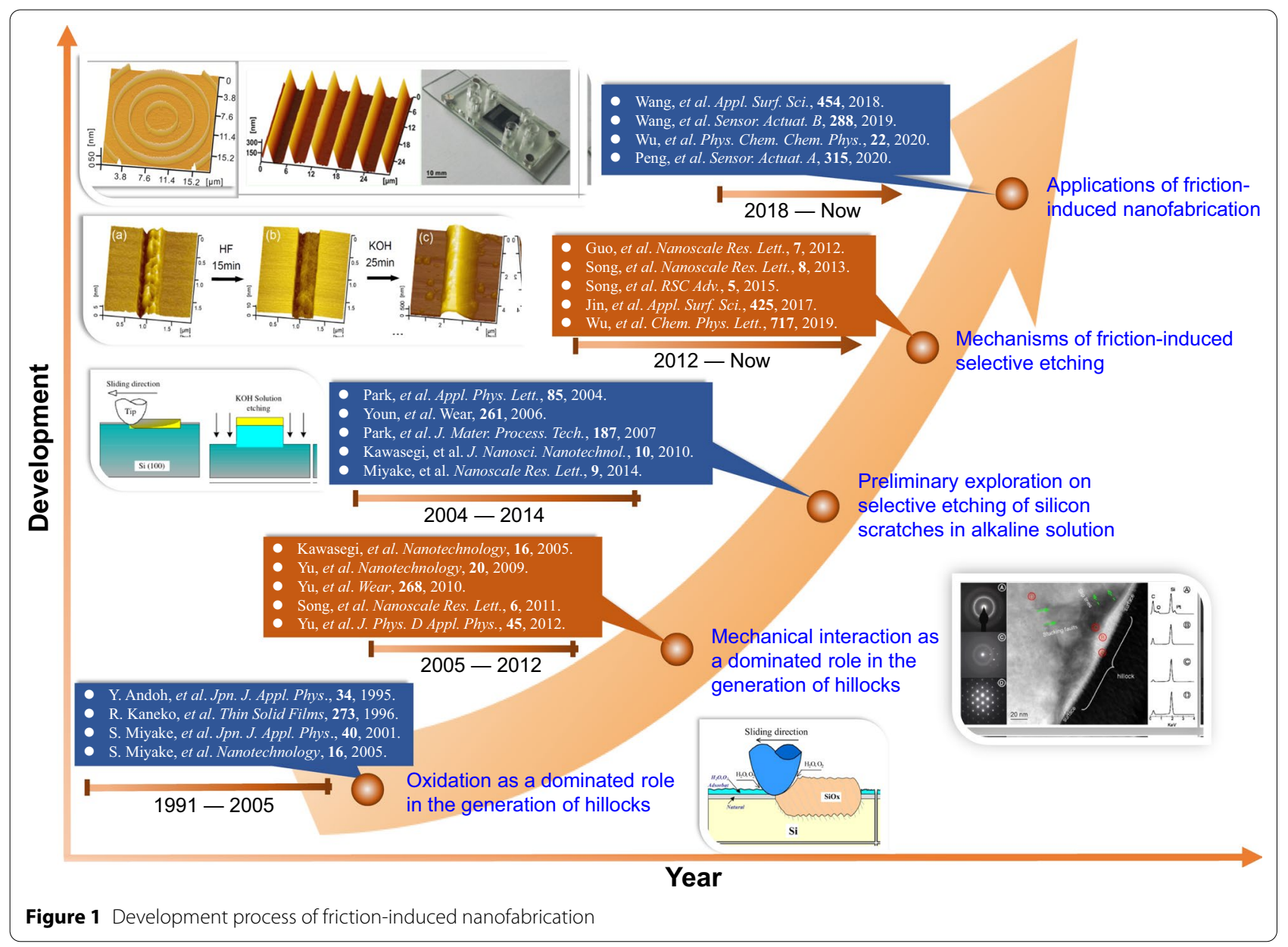

(PMMA) film with AFM-based ultrasonic vibrationassisted approach, which was operated in constant height control mode [29]. Two methods, i.e., the operations under vector mode and raster scan mode, were adopted to design stereoscopic features to be machined. Relatively simple features, such as stair-like nanostructure with five steps was successfully fabricated in vector mode, while complex nanostructure (e.g., Pyramid 3D structure and the profile of Steve Jobs) were successfully fabricated in raster scan mode from bitmap images. This route can reduce effectively tip wear and enlarge the size of fabricated nanostructures.

In contrast with static plowing lithography, DPL can effectively avoid edge irregularities on fabricated nanostructures, and hence DPL is becoming a promising and reliable approach for fabricating complex nanostructures [30]. He et al. [31] applied DPL with an AFM to fabricate periodic nanostructures on PMMA film (Figure 2b). In their study, a silicon tip was employed for scratching PMMA surface under various scanning angles in tapping mode, and high-quality periodic nanodot arrays were obtained through optimizing tip traces, and the density of the nanodots achieved could be as high as $1.9 \times 10^{9}$ nanodots per $\mathrm{mm}^{2}$. Similarity, DPL technique was also employed to fabricate various structures on graphene film surface (Figure 2c), extending the possibilities for AFM-based manipulation of graphene [32]. Moreover, it was found that the lithography significantly depended on the applied force. With moderate forces, AFM tip only deformed the graphene and generated local strain. In contrast, with sufficiently large forces the AFM tip can hook the graphene and then pull it, thus cutting the graphene along the direction of the tip motion. Further characterization in conjunction with electric force microscopy, Kelvin probe force microscopy and conductive AFM demonstrated that DPL process cannot change local electrical properties of the patterned graphene structure.

\subsection{Direct Nanofabrication of Protrusive Hillocks}

When scratched by a diamond tip under given conditions, hillock-like nanostructures can be produced on monocrystalline silicon surface [33]. The investigation of protrusive hillock formation could be traced back to 
(a)
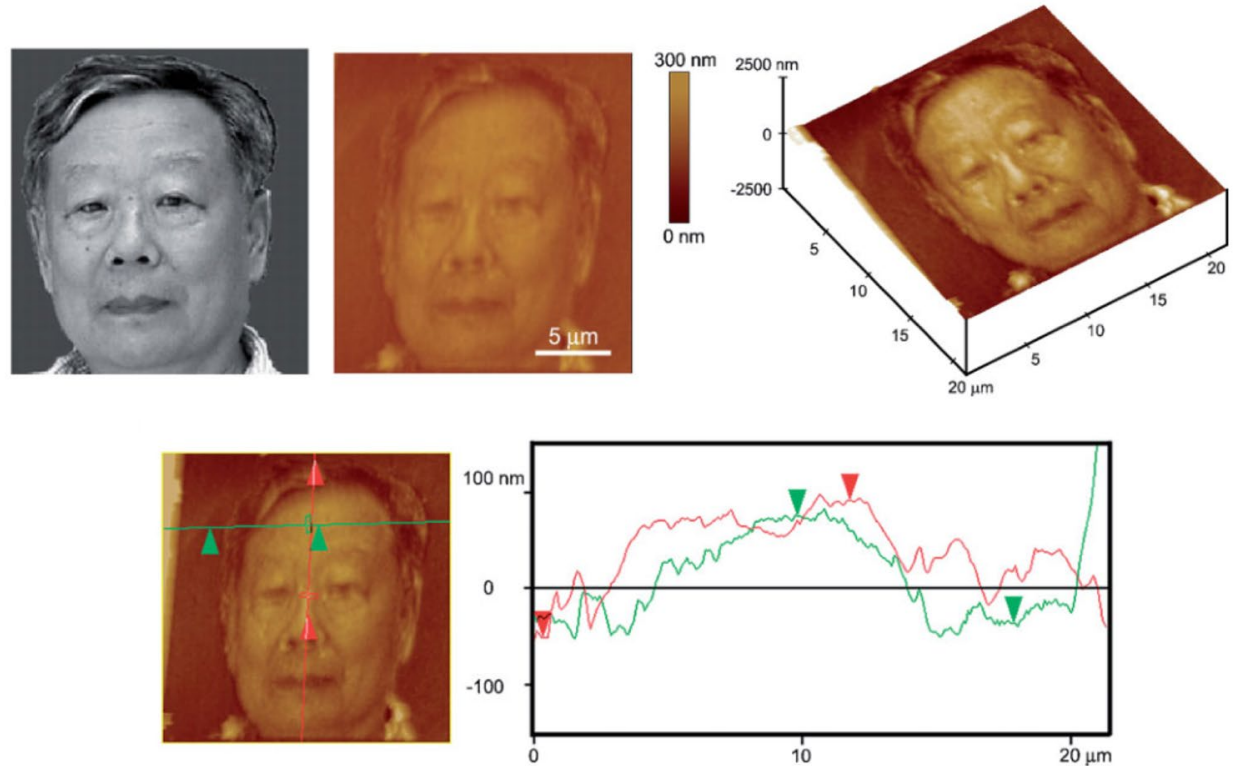

(b)
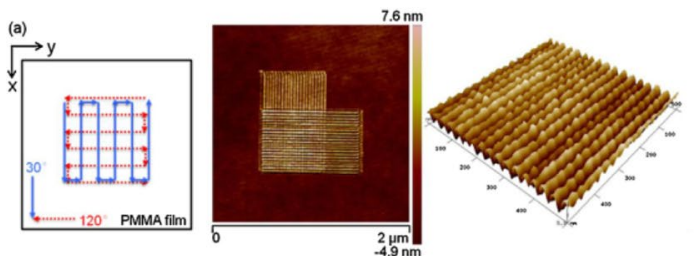

(c)

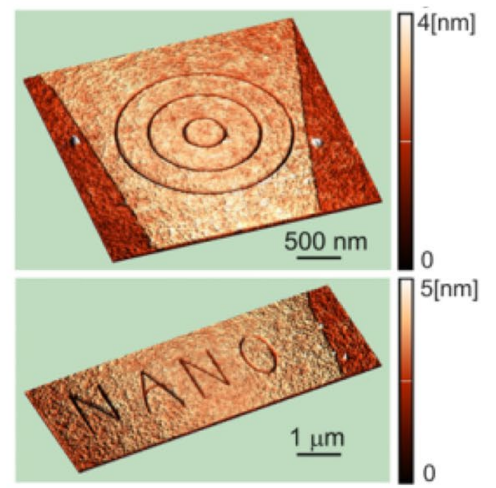

Figure 2 Nanopatterning on material surfaces through mechanical removal by scanning probe: (a) Fabrication of human face nanostructure on Al surface under contact mode [28], (b)-(c) Fabrication of nanostructures on PMMA (Figure 2b) and graphene film (c) using dynamic plowing lithography based on AFM [31,32]

the microwear on polymer surface in 1990s [34, 35]. In 1990s, Kaneko et al. [33] proposed two kinds of microwear processes of materials. The first one is to form directly grooves on material surface, and the other is to produce upheaval firstly resulting from plastic deformation and tribo-chemistry, followed by the formation of grooves structure. To some extent, friction-induced hillocks could be a kind of damages in early stage of material wear. However, Yu et al. [36] found that protrusive hillocks can hardly be formed in an indentation test or in a line-scratch experiment where the scratch distance was too small for diamond tip slipping, but often appeared in a long-distance scratch process, as shown in Figure 3. The sliding and friction appear to be necessary conditions for the generation of hillocks, and this process can be viewed as friction-induced nanofabrication [24].

\subsubsection{Pressure Threshold for the Formation of Friction-Induced Hillocks on Monocrystalline Silicon}

The scratching by a sharp AFM tip can usually lead to material remove and thereby form nano-deep grooves, which is a typical top-down processing [37]. However, protrusive hillocks can also be produced along the scratching line under some given conditions. The formation of grooves or hillocks depends strongly on tip radius and normal load, namely that the contact stress between AFM tip and sample determines the ultimate appearance of hillocks in scratching [24]. Therefore, it is necessary to 


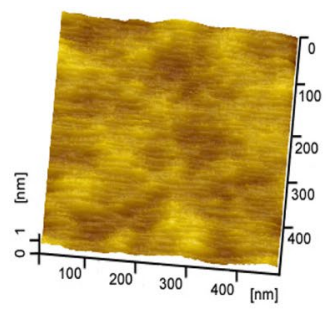

$D=0.5 \mathrm{~nm}$

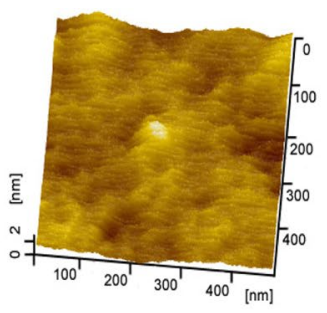

$D=5 \mathrm{~nm}$

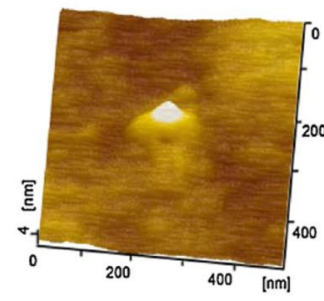

$D=12.5 \mathrm{~nm}$

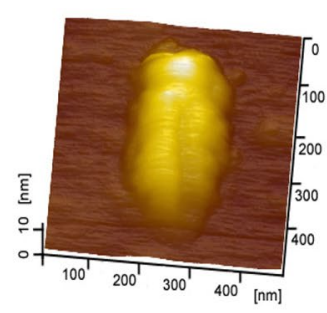

$D=100 \mathrm{~nm}$

Figure 3 AFM images of the scars on Si(100) surface after scratching at various displacement amplitudes D [36]

detect the threshold load for the transition from protrusion to material removal in the friction process.

Under light normal load contacting, elastic deformation can occur on monocrystalline silicon surface [38]. Considering the applied normal load and tip radius in the scratching, the contact pressure threshold can be estimated based on Hertz elastic contact theory [24, 39]. The contact pressure threshold of hillock formation on silicon was $\sim 11 \mathrm{GPa}$ (Figure 4 ), which was approximately equal to the calculated value $11.3 \mathrm{GPa}$ by the third strength theory (maximum shear stress theory), and was also close to the value 11-13 GPa [40-42] of Vickers hardness of monocrystalline silicon. As a result, the determination of contact pressure threshold provides the basis for the selection of normal load for a diamond tip with known radius for producing friction-induced hillocks.

\subsubsection{Processing Parameters-Dependent Formation of Friction-Induced Hillocks}

Aside from applied normal loads, the fabrication of friction-induced hillocks is also strongly dependent on scratching cycle numbers, crystal orientations, surface oxide thickness, processing atmosphere, and so on, as demonstrated in Figure 5a [24-26, 43]. Under a given processing condition, the height of the friction-induced hillocks increased with the applied normal load or scratching cycle numbers. Under the same loading condition, the hillock produced on $\mathrm{Si}(100)$ surface is the highest, while that produced on $\mathrm{Si}(111)$ surface is the lowest [43]. Further analysis deduced that the difference in the hillock height on various crystal planes may correlate with surface mechanical properties and bond structures. The formation of friction-induced hillocks was expected to be dependent on configures of dangling bonds, inplane bonds, and back bonds on different crystal planes [44-46]. It is also of great interest to note that the height of friction-induced hillocks decreased with the increase of sliding velocity $(10-1000 \mu \mathrm{m} / \mathrm{s})$ both in air and in vacuum [26]. The sliding velocity-dependent hillock formation is related to energy dissipation during tip scratching [26], which would be addressed in the following section.

Especially, in 2010, Yu et al. reported the formation of friction-induced hillocks in vacuum for the first time, and the normal load-and scratching numbers-dependent

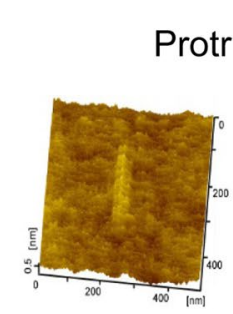

$0.5 \mu \mathrm{N}$

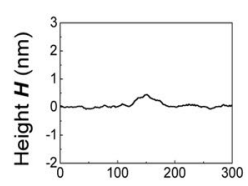

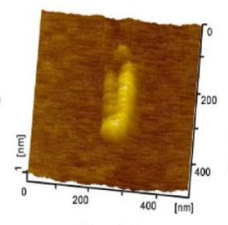

$5 \mu \mathrm{N}$
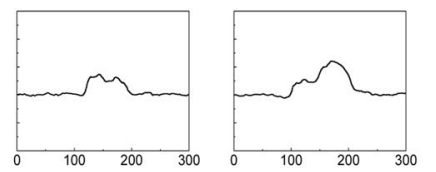

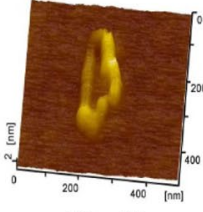

$10 \mu \mathrm{N}$

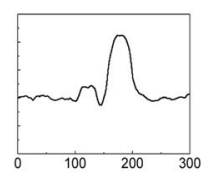

Distance $(\mathrm{nm})$
Sunken grooves

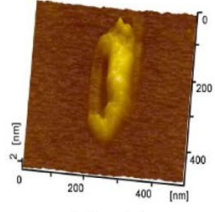

$15 \mu \mathrm{N}$

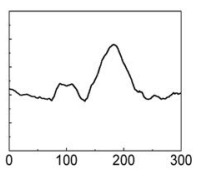

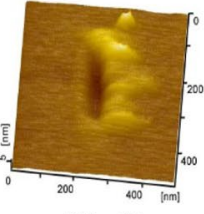

$30 \mu \mathrm{N}$

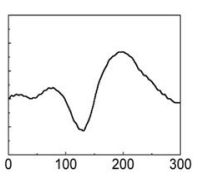

Figure 4 AFM images (top) and cross-sectional profiles (bottom) of the scratches on Si(100) surface after line-scratching under various normal load $F_{\mathrm{n}}[36]$ (The diamond tip for the test has tip radius of about $0.15 \mu \mathrm{m}, \mathrm{D}=100 \mathrm{~nm}$ and $N=100$ ) 

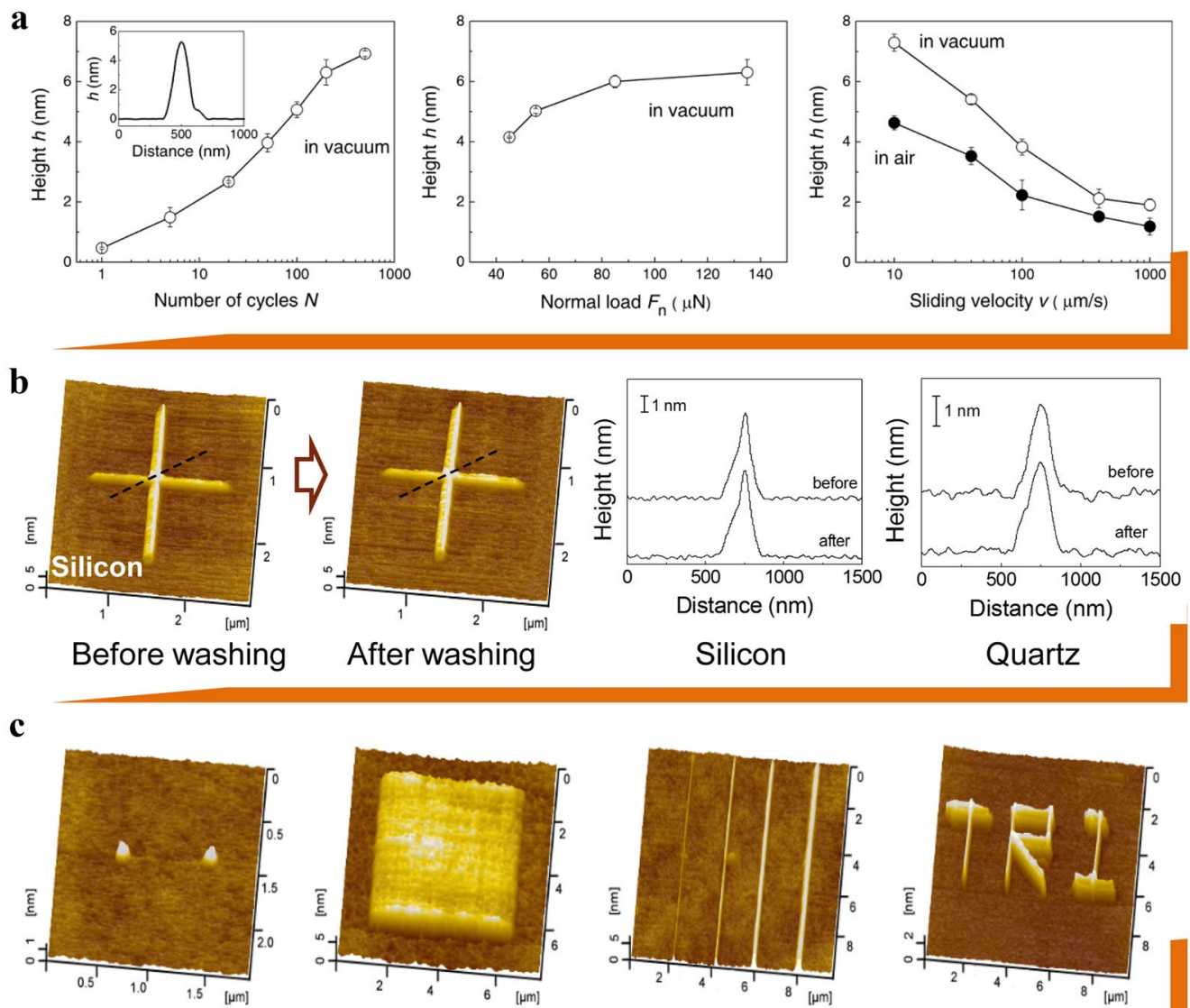

Figure 5 (a) Hillock height $h$ as a function of number of scratching cycles $N$, applied normal load $F_{n}$, and sliding velocity $v$ [26]. (b) AFM images and corresponding cross-sectional profiles of friction-induced hillocks on silicon and quartz surfaces before and after washing [47]. (c) AFM images of various protrusive nanostructures (i.e., nanodots, surface isolated mesa, nanoline array, and pattern of "TRI") produced by reciprocating scratching on silicon surface [24]

hillock formation was studied systematically with an AFM, as shown in Figure 5a [25]. Based on the vacuum test, it is strongly suggested that the friction-induced hillock could be composed of deformed silicon, such as amorphous silicon and dislocations, but not only silicon oxides. In other words, mechanical interaction should play a significant role in the formation of friction-induced hillocks.

The mechanical stability of nanostructures directly determines their reliability during the service. After the friction-induced hillocks on silicon and quartz surfaces were ultrasonically cleaned in deionized water, hardly any change in surface topography can be noticed through comparing cross-sectional profiles (Figure 5b), demonstrating that prepared friction-induced hillocks possessed excellent mechanical stability [47]. Also, nanoindentation tests indicated that elastic modulus of friction-induced hillocks on silicon and quartz surfaces was a little lower than that of their substrates. Nevertheless, nanoscratch tests confirmed that friction-induced hillocks on silicon and quartz surfaces can withstand typical contact in dynamic MEMS [47-49]. In view of this, frictioninduced hillocks presented good mechanical strength and stability. Friction-induced nanofabrication of protrusive hillocks can be easily applied for producing various nanostructures on silicon, quartz and glass surfaces. Figure $5 \mathrm{c}$ displays surface topographies of various protrusive hillock nanostructures, i.e., nanodots, surface isolated mesa, nanoline array, and pattern of "TRI", produced by reciprocating scratching of silicon surfaces, confirming an excellent fabrication ability.

\subsubsection{Mechanism for Friction-Induced Hillock Formation}

The formation mechanism of the hillocks has puzzled people for a long time, and many efforts have been devoted on revealing the formation mechanism of the friction-induced hillocks from 1990s [50]. Miyake et al. $[51,52]$ speculated that the generation process of 


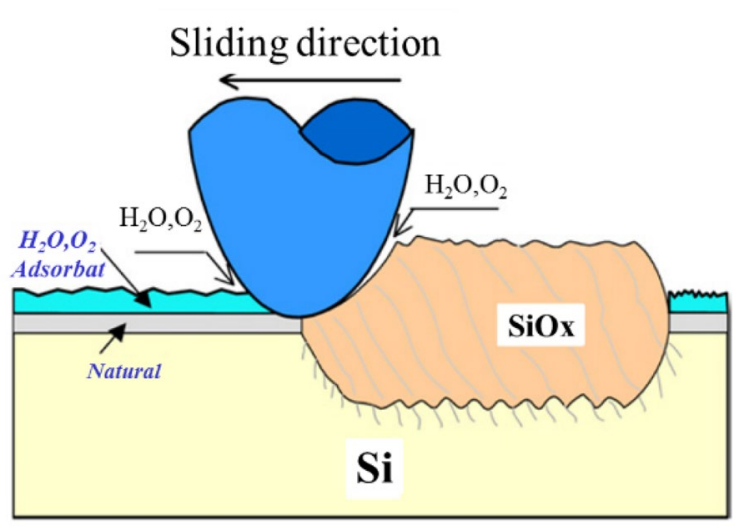

Figure 6 Oxidation-dominated generation of protuberances on silicon surface speculated by Miyake et al. [52]

protuberances was ascribed to local atomic destruction of chemical bonds that occurs with concentrated stress. Specifically, the reaction of damaged silicon with oxygen and water was enhanced by the sliding, forming silicon oxide or silicon hydroxide (Figure 6). Since then, it can be accepted that the upheaval formed on silicon surface was the results of the oxidation with the participation of adsorbed air and water [53-55]. Unlike the past decade, however, Youn and Kang [56] investigated the effect of single-point diamond machining conditions on the deformation behavior of monocrystalline silicon, and they suggested that the plastic deformation in silicon may contribute to the formation of surface hillocks during scratching. Meanwhile, considering the formation of friction-induced hillock in vacuum, the oxidization should not the only contributor to the hillock height.

To detect any potential chemical reactions during the scratching of $\mathrm{Si}(100)$ surface, friction-induced hillocks with a height of $\sim 3.5 \mathrm{~nm}$ were prepared on $\mathrm{Si}(100)$ surface under area-scanning mode [25]. By scanning X-ray microprobe detecting, the atomic number ratio of $\mathrm{O} / \mathrm{Si}$ increases from 0.60 on the original $\mathrm{Si}(100)$ surface to 0.68 on the hillocks created in vacuum and 0.97 on the hillocks created in atmosphere. The atomic concentration of oxygen was also detected as a function of the depth by a scanning Auger nanoprobe on the hillocks and original $\mathrm{Si}(100)$ surface, respectively. As shown in Figure 7, the thickness of oxidation layer was $2.0 \mathrm{~nm}$ on the hillock produced in atmosphere, while the hillock created in vacuum has a $1.7 \mathrm{~nm}$ oxidation layer. When deducting $0.5 \mathrm{~nm}$ native oxide on original silicon surface, the oxidation layer formed during the scratching should be $1.5 \mathrm{~nm}$ in atmosphere and $1.2 \mathrm{~nm}$ in vacuum, which were much smaller than the height of the detected hillocks $(\sim 3.5 \mathrm{~nm})$. In addition, friction-induced hillock can

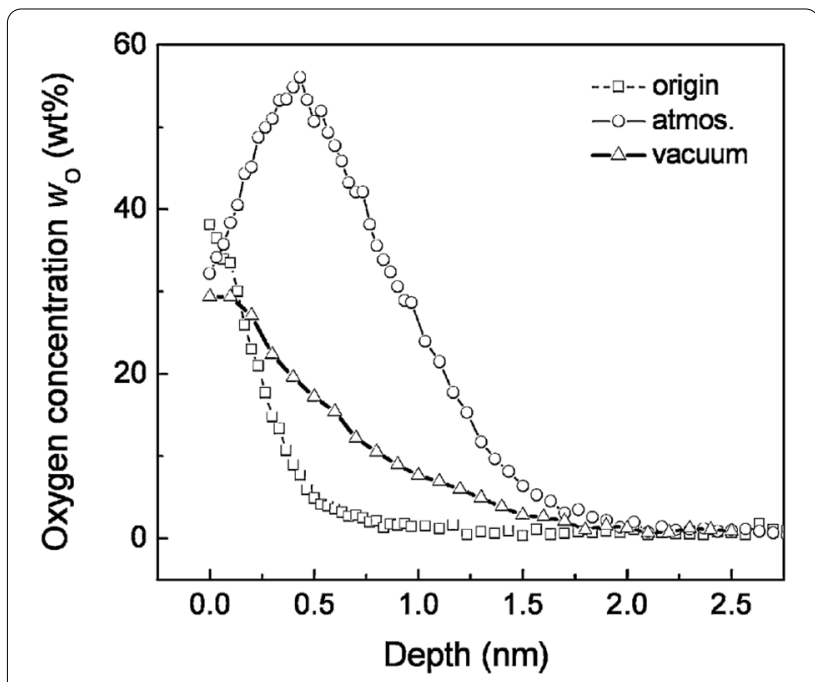

Figure 7 Comparison of atomic concentration of oxygen in wt $\%$ measured by a scanning Auger nanoprobe on the original Si(100) surface, on the hillocks created in atmosphere and in vacuum [25]

also be produced on glass and quartz surfaces, respectively [25]. Such materials are composed of oxidations or stable compounds, which could be hardly oxidized in scratching, confirming that mechanical interaction, i.e., structural deformation, should be responsible for the formation of friction-induced hillocks.

The microstructure of the hillock with a height of $\sim 7$ $\mathrm{nm}$ was analyzed by cross-sectional transmission electron microscope (XTEM) [24]. As shown in Figure 8, a triangular zone consisting of a bright top layer and a relatively dark bottom was detected from the cross section, which was prepared by focused ion beam (FIB) cutting. Selected area diffraction (SAD) patterns confirmed that the bright top case is amorphous silicon with a maximum thickness of $\sim 20 \mathrm{~nm}$ (Figure 8A; the sharp rings was contributed from protective Pt layer). Beneath the amorphous layer, a deformed zone with a maximum depth of $\sim 80 \mathrm{~nm}$ was found with distorted crystal matrix (Figure $8 \mathrm{C}$ ), which is quite different from monocrystalline silicon (Figure 8D). Many crystal defects were observed in the deformed dark zone, such as slip lines and stacking faults.

As shown in the right panel of Figure 8, from EDX analysis on the XTEM sample, both oxygen and silicon were detected form the hillock surface area (A), suggesting that $\mathrm{SiO}_{2}$ may have formed on silicon surface when scratched by the AFM tip. However, no obvious oxygen peak could be detected from the interior of the amorphous zone (B), severe deformed zone $(\mathrm{C})$ and original monocrystalline silicon (D). These results are consistent with the Auger analysis of oxygen concentration in Figure 7 . It is confirmed that oxidation reaction occurred 

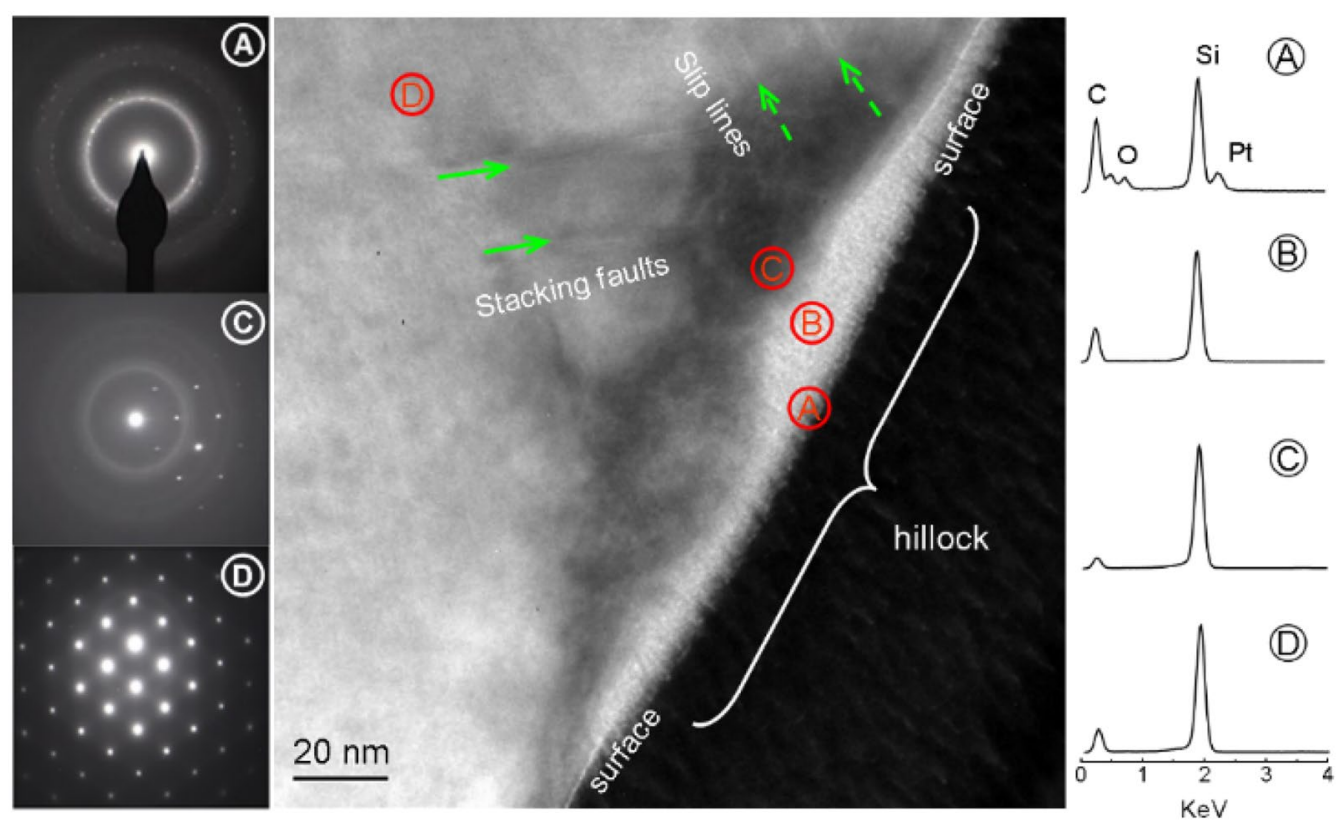

Figure 8 XTEM image showing the cross-sectional structure of a typical hillock formed on Si(100) surface [24] (The left pictures show the SAD patterns from areas: A—amorphous rings for the hillock (sharp rings from Pt), C—deformed and amorphous silicon, and D—substrate silicon ( $b=$ 110). The right pictures show the EDX spectra from different marked areas of the cross-section)

only at near surface of the hillock and the thickness of oxide layer was much smaller than that of the mechanically interacted layer (amorphous silicon and deformed silicon). Therefore, it is logically deduced that even if both tribochemical reaction (i.e., formation of silicon oxide) and mechanical interaction may have contributed to the generation of friction-induced hillocks, the latter (i.e., formation of amorphous structure and crystal defects) should play a dominating role to induce the hillock formation on silicon surface. Such a conclusion can be further verified by the formation of hillocks in vacuum $\left(6.7 \times 10^{-4} \mathrm{~Pa}\right)$, on glass and quartz $\left(\mathrm{SiO}_{2}\right)$ surfaces $[24$, 48], where it is impossible for oxidizing to occur.

For deeper understanding the formation of frictioninduced hillocks on monocrystalline silicon, high-resolution transmission electron microscope (HRTEM) were performed for detecting cross-sectional microstructures of the hillocks produced under various sliding velocity [26]. As shown in Figure 9a, only a thick amorphous layer was detected on cross section of the hillock produced at a low speed of $10 \mu \mathrm{m} / \mathrm{s}$ in vacuum. By contrast, a thin amorphous layer and severe matrix deformation, including slip bands and stacking faults, were observed on cross section of the hillock produced at a high speed of 1000 $\mu \mathrm{m} / \mathrm{s}$, as shown in Figure $9 \mathrm{~b}, \mathrm{c}$, and d. These slip bands and stacking faults are aligned on the preferential slip planes $\{111\}$, which is predicted by molecular dynamics analysis [57]. It is speculated that more energy is
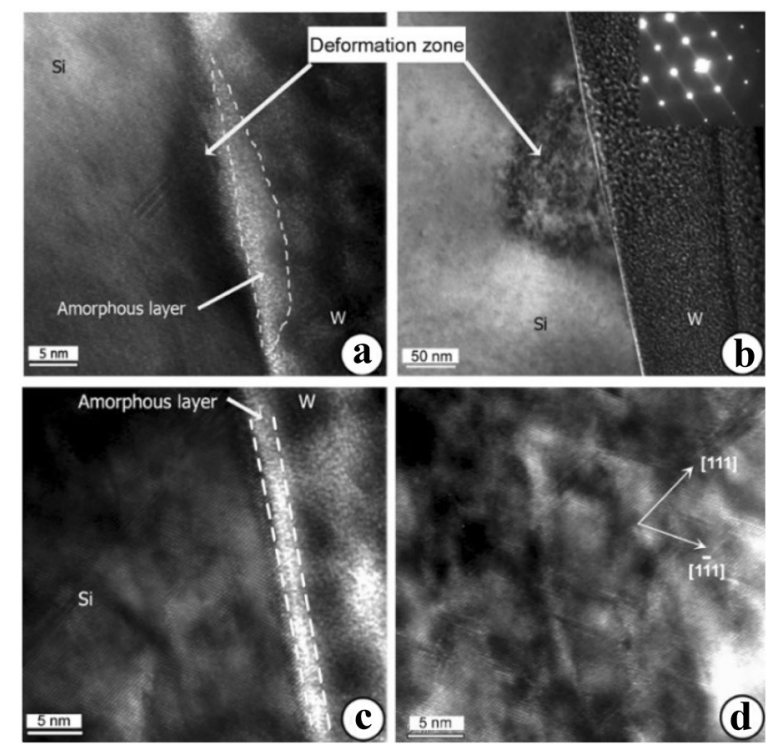

Figure 9 XTEM microstructure of the silicon hillocks created at various sliding speeds in vacuum [26]: (a) XTEM microstructures of the hillock created at $v=10 \mu \mathrm{m} / \mathrm{s}$, and (b) $v=1000 \mu \mathrm{m} / \mathrm{s}$. The inset SAD pattern in (b) was taken from the area under the hillock, (c) and (d) high resolution TEM microstructures of amorphous layer and deformation zone in (b) 
speculated to be dissipated in facilitating a-Si formation during scratching at low speed. In contrast, the amorphization is difficult to occur within the short interaction time, and hence most of the input energy is dissipated in the formation of deformed silicon during high-speed sliding [47].

As a result, a thicker amorphous layer corresponds with a higher hillock, and mechanical interaction through the generation of amorphous structure is the key contributor to hillock formation on silicon surface. Based on the detection of oxygen concentration by a scanning Auger nanoprobe in Figure 7, oxidation-induced increment of hillock height during scratching was estimated, and the oxidation contribution to the total height of the detected hillocks is less than 23\% [25]. Considering that the scratched area is partly oxidized (Figure 7), later estimation indicated the contribution from oxidation to the height of the hillock is as less as $3.1 \%$ in vacuum and $11.3 \%$ in air [47]. Moreover, the increase of scratching cycles can lead to further amorphization or break of $\mathrm{Si}-\mathrm{Si}$ bonds [56], yielding higher hillocks. In addition, when the normal load increased, more silicon matrix was amorphized and thereby higher friction-induced hillocks were produced. The localized amorphous structure was expected to present lower density than crystal silicon, and hence piled up to form a hillock [58]. Similarly, the amorphization-dominated interaction may be the main contributor for the formation of friction-induced hillocks on other materials, such as glass and quartz [25, 47, 48]. It should be noted that fused silica and glass are typical amorphous materials, and the surface hillocks may be resulted from by the amorphization of short-range ordered structures. However, the details for the generation of friction-induced hillocks remains to be further clarified, which can start from the investigation on energy threshold, crystal transition paths, amorphous transformation, and so on.

\section{Friction-Induced Selective Etching on Silicon with Scratch Mask}

The fabrication based on scanning probe scratching faces many challenges. For instance, the aspect ratio of fabricated structures was relatively low, and the scratching process would easily cause material damage and tip wear, impacting subsequent applications of fabricated structures. In view of this, combing SPM scratching and wet chemical etching, friction-induced selective etching [27], which is also called tribo-nanolithography or scanning mechano-chemical probe lithography [59], was developed for fabricating micro/nanostructures on material surface. This route could meet SPL technical challenges in wear debris, tool wear, and substrate damage to some extent.

Many scholars were devoted to investigating the feasibility of this fabrication technique and revealing involved mechanisms. Youn et al. [56] investigated the effect of single-point diamond machining conditions, e.g., tip orientation and normal load, on both deformation behavior and chemical properties of monocrystalline silicon through nanoscratch and $\mathrm{KOH}$ solution etching. It was demonstrated that the orientation of Berkovich indent tip showed a significant effect on etching behaviors of silicon in $\mathrm{KOH}$ solution (Figure 10), and higher normal load resulted in the increase of etch-mask ability of the mechanically affected layer. Besides, if the sample surface is non-flat, the tip can adjust to surface variations and machines a constant-depth nanostructure. Sung et al. [60] successfully applied SPL technique to fabricate patterns on a tapered 1-hexadecanethiol (HDT)/Ag surface with a height and width of $2.5 \mu \mathrm{m}$ and $100 \mu \mathrm{m}$, respectively (Figure 11a). After being etched in oxygenated cyanide solution for $20 \mathrm{~s}$, the pattern was successfully transferred from HDT to the Ag surface because the HDT can act a mask against the etching (Figure 11b).

Also, some special structures, such as slop and a structure with vertical side walls, can be realized by tip scratching and subsequent selective etching. Park et al. [61] used this technique to fabricate a protrusive hillock with a slope (Figure 12a). In their study, a diamond tip was used for scratching silicon surface with a given pitch in $\mathrm{KOH}$ solution, and the generation of the structure was attributed that the scratched regions can act a mask against the etching while unscratched surface continued to dissolve. Moreover, the inclination $(\alpha)$ of the protrusive structures was demonstrated to present strong correlation with scratching speed in $y$ direction, but does not tightly correlate with scan pitch, speed in the $\mathrm{x}$ direction, and normal force. Kawasegi et al. [62] applied this technique to fabricate high-aspect-ratio structures on silicon surface (Figure 12b). Through scratching along $<112>$ direction on $\mathrm{Si}(110)$ surface and subsequent selective etching, a structure with vertical side walls was fabricated, and the width and height of the structures were dominated by normal load and scratching number. This fabrication technique was becoming a promising candidate for the fabrication of Si-based functional structures and/or devices.

\subsection{Mechanisms for Scratch Masking in Friction-Induced Selective Etching}

In past study, researchers speculated that oxygen-rich layer in scratched regions can resist etchant etching, resulting in the formation of protrusive nanostructures on silicon surface [51, 52, 62, 63]. However, it is noted 


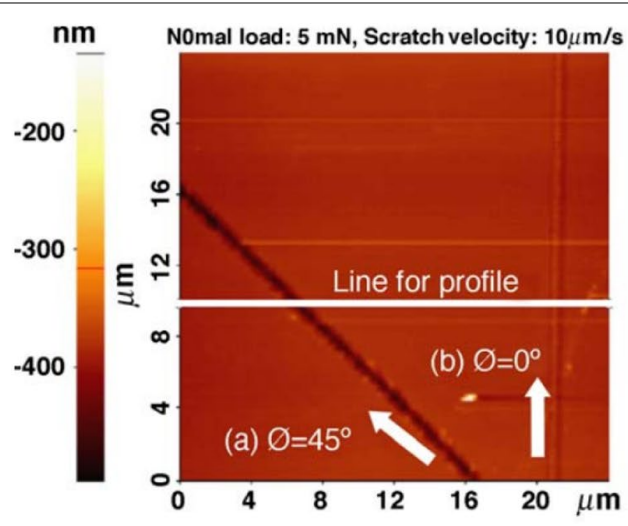

Surface profile $(\mathrm{nm})$

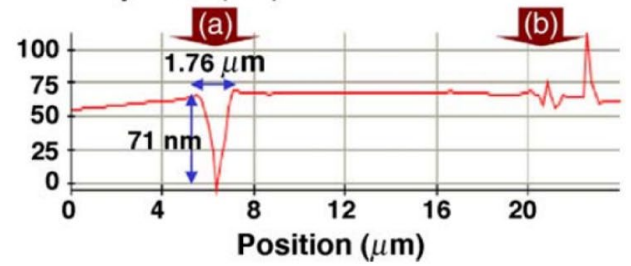

a

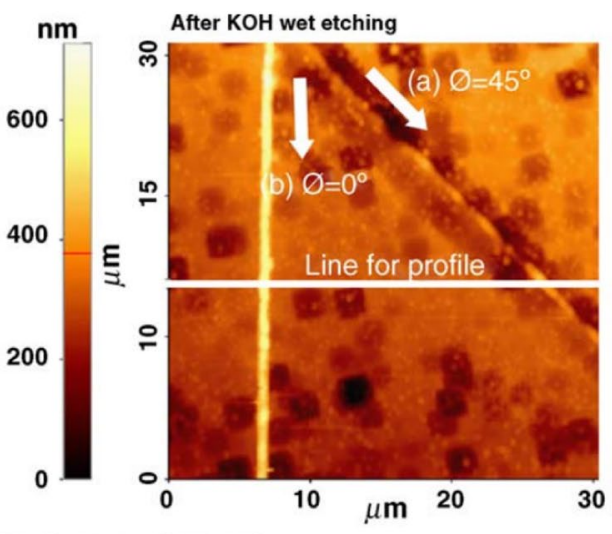

Surface profile $(\mathrm{nm})$

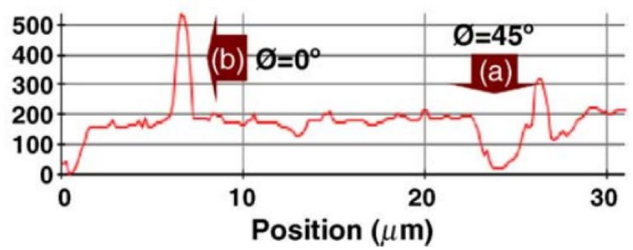

b

Figure 10 AFM images (top) and corresponding cross-sectional profiles (bottom) of nanoscratches created with different tip direction $\left(\varphi=0^{\circ}, 45^{\circ}\right)$ [56]: (a) before etching; (b) after 20 wt.\% $\mathrm{KOH}$-etching for $18 \mathrm{~min}$
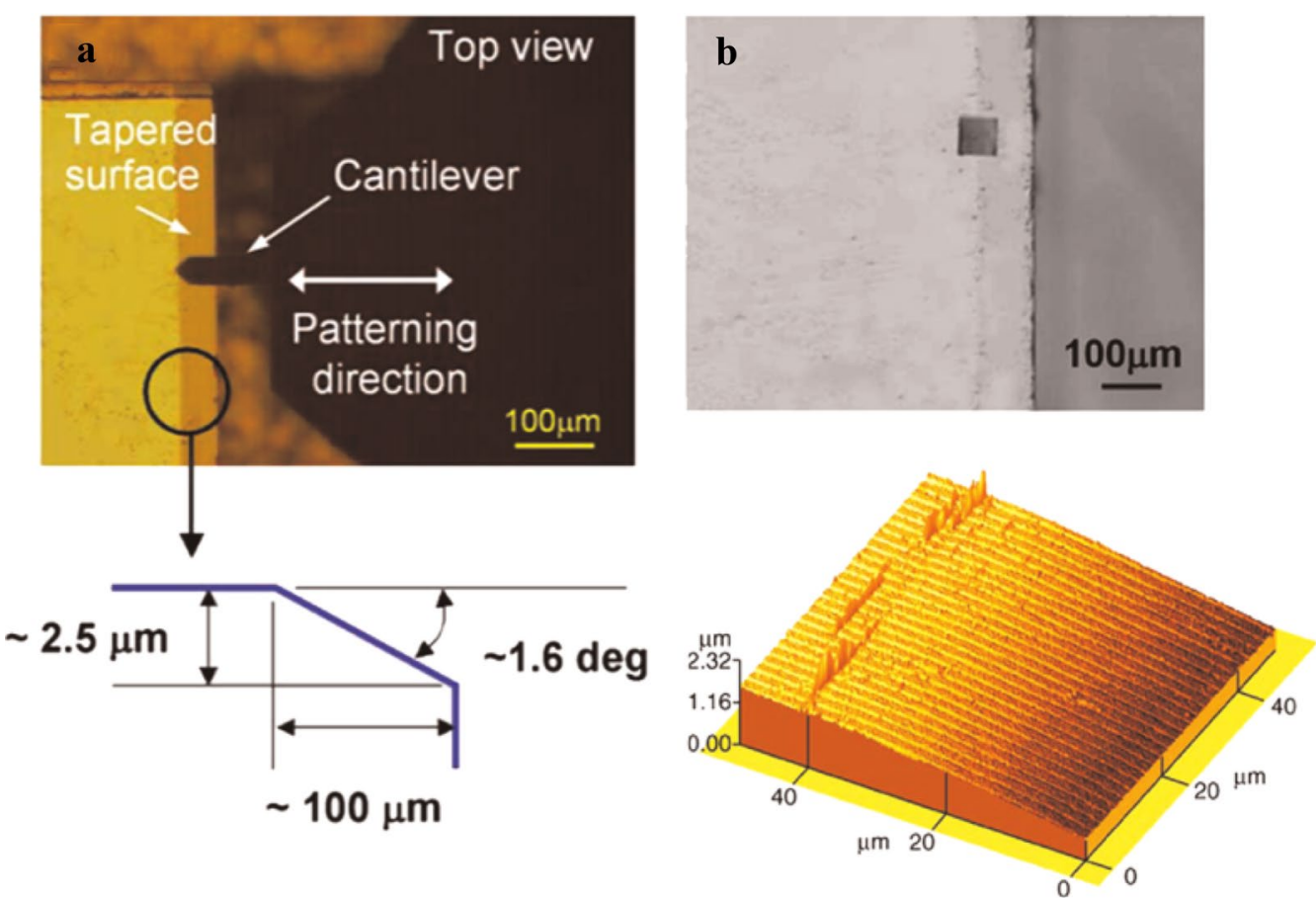

Figure 11 Nanopatterns fabricated on an inclined HDT/Ag surface through a diamond-coated tip scratching and subsequent chemical etching [60] 

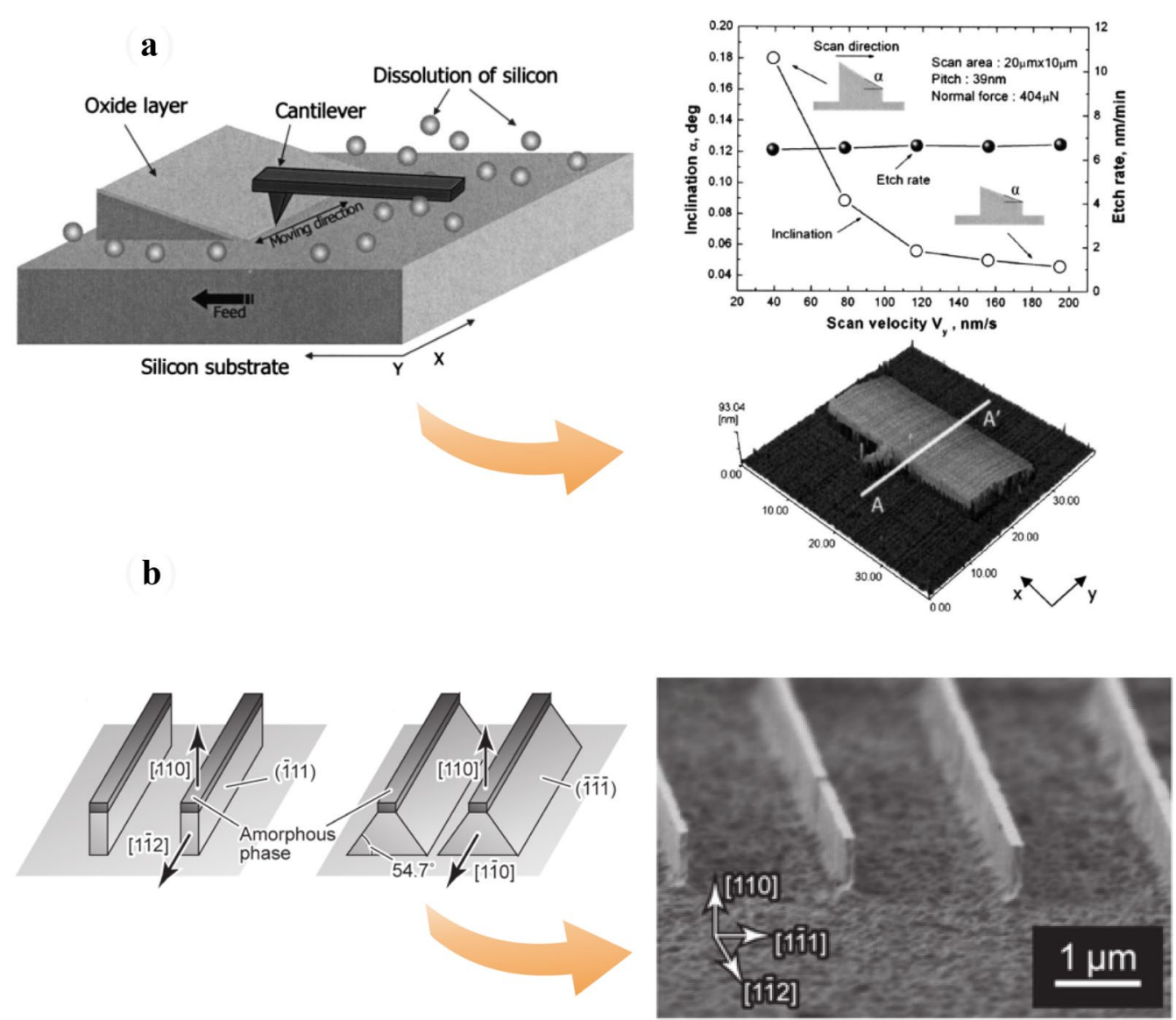

Figure 12 Fabrication of silicon nanostructures through scratching silicon surfaces with a diamond tip followed by subsequent wet etching [61, 62]: (a) Schematic diagram showing the sliding process in $\mathrm{KOH}$ solution. The right panel displayed AFM topography of fabricated structures, and the correlation of inclination (a) of the protrusive structures and scratching speed in the $y$ direction $\left(V_{y}\right)$. (b) Schematic diagram showing the shape of the structure fabricated by the scratching along $\langle 112\rangle$ and $\langle 110\rangle$ directions. The right panel displayed SEM image of high-aspect-ratio structures fabricated by the scratching along $<112>$ direction and subsequent etching in $\mathrm{KOH}$ solution

that mechanical scratch-induced subsurface deformation not only consists of oxidation layer, but also contains amorphous silicon and deformed silicon lattice [64]. Accordingly, the newly formed protrusive nanostructures after the etching cannot be attributed completely to the anti-etching performance of superficial oxygen-rich layer in scratched regions. Hence, some researches were dedicated to uncovering selective etching mechanism of scratched silicon surface in various etchants, e.g., $\mathrm{KOH}$, TMAH and $\mathrm{HF} / \mathrm{HNO}_{3}$.

To clarify selective etching mechanism for scratched regions in $\mathrm{KOH}$ solution, superficial oxidation layer of a groove-shaped scratch was removed completely by HF solution, and hillock structures were still observed after the HF-treated scratch was further etched by $\mathrm{KOH}$ solution (Figure 13) [27]. Such a result showed that the deformed silicon lattice in the scratched area can act a mask against $\mathrm{KOH}$ etching. Based on the electrochemical model proposed by Seidel et al. [65], it is deduced that the selective etching behavior was mainly attributed to the differences on the dangling bond density between scratched regions and original silicon surface. When silicon surface was scratched, the atomic arrangement of amorphous silicon could become out of order, and the average dangling bond density in scratched regions was lower than original Si surface [66]. Accordingly, etching rate in scratched regions was slow down, resulting in the formation of hillock nanostructures. Similar performance and mechanisms were assigned on the friction-induced selective etching on silicon surface when TMAH solution was employed [67].

The mixture of $\mathrm{HF}$ and $\mathrm{HNO}_{3}$ solution was isotropic system for processing silicon wafers, and was good compatibility with on-chip circuitry. However, etching profile was limited by isotropic dissolution of silicon, which impedes its practical applications for the fabrication 

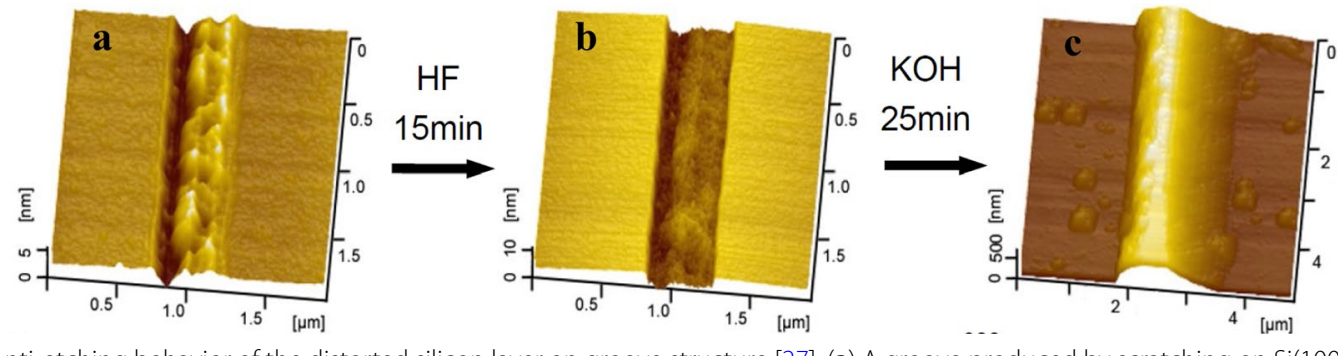

Figure 13 Anti-etching behavior of the distorted silicon layer on groove structure [27]: (a) A groove produced by scratching on Si(100) surface. (b) AFM image of the scratch after being etched in $10 \mathrm{wt} . \% \mathrm{HF}$ solution for $15 \mathrm{~min}$ to remove surface oxidation layer. (c) AFM image of the scratch after being etched in $20 \mathrm{wt} . \% \mathrm{KOH}+$ IPA solution for $25 \mathrm{~min}$

of nano-sized functional devices [68]. Fortunately, HF/ $\mathrm{HNO}_{3}$ mixture presented excellent selective etching performance on scratched regions and thereby fabricated high-quality nanochannels [69]. It is noted that the depth of groove-shaped scratch can be deepen after being etched by HF solution, but its inner surface roughness was relatively high and the depth of nanochannels was lower than that by $\mathrm{HF} / \mathrm{HNO}_{3}$ mixture. In contrast, when the etchant was replaced by $\mathrm{HNO}_{3}$ solution, selective etching was hardly detected. Hence, the excellent selective etching ability for scratched regions in $\mathrm{HF} / \mathrm{HNO}_{3}$ mixture was attributed to the synergistic effect of $\mathrm{HF}$ and $\mathrm{HNO}_{3}$ solution. To further clarify the selective etching mechanism, cross-sectional microstructures of scratched regions before and after being etched by $\mathrm{HF}$ and $\mathrm{HF} /$ $\mathrm{HNO}_{3}$ mixture was observed by HRTEM (Figure 14). The results indicated that $\mathrm{HF} / \mathrm{HNO}_{3}$ mixture can remove completely all scratch-induced subsurface defects including amorphous and deformed silicon, but HF solution can only etch amorphous and is powerless for deformed silicon. Accordingly, Wang et al attributed the peculiar selective etching phenomenon to the coupling action of three factors, i.e., lattice defects, low activation energy and excess holes [69]. As main subsurface defects, amorphous silicon and dislocations widen lattices and silicon atom spacing, providing a shortcut for etchant molecule diffusing to reaction interface. Moreover, input mechanical energy during scratching process facilitated electrons transition and emission, leading to a relatively high holes density in scratched regions, which can be beneficial for rapid dissolution of scratched regions.

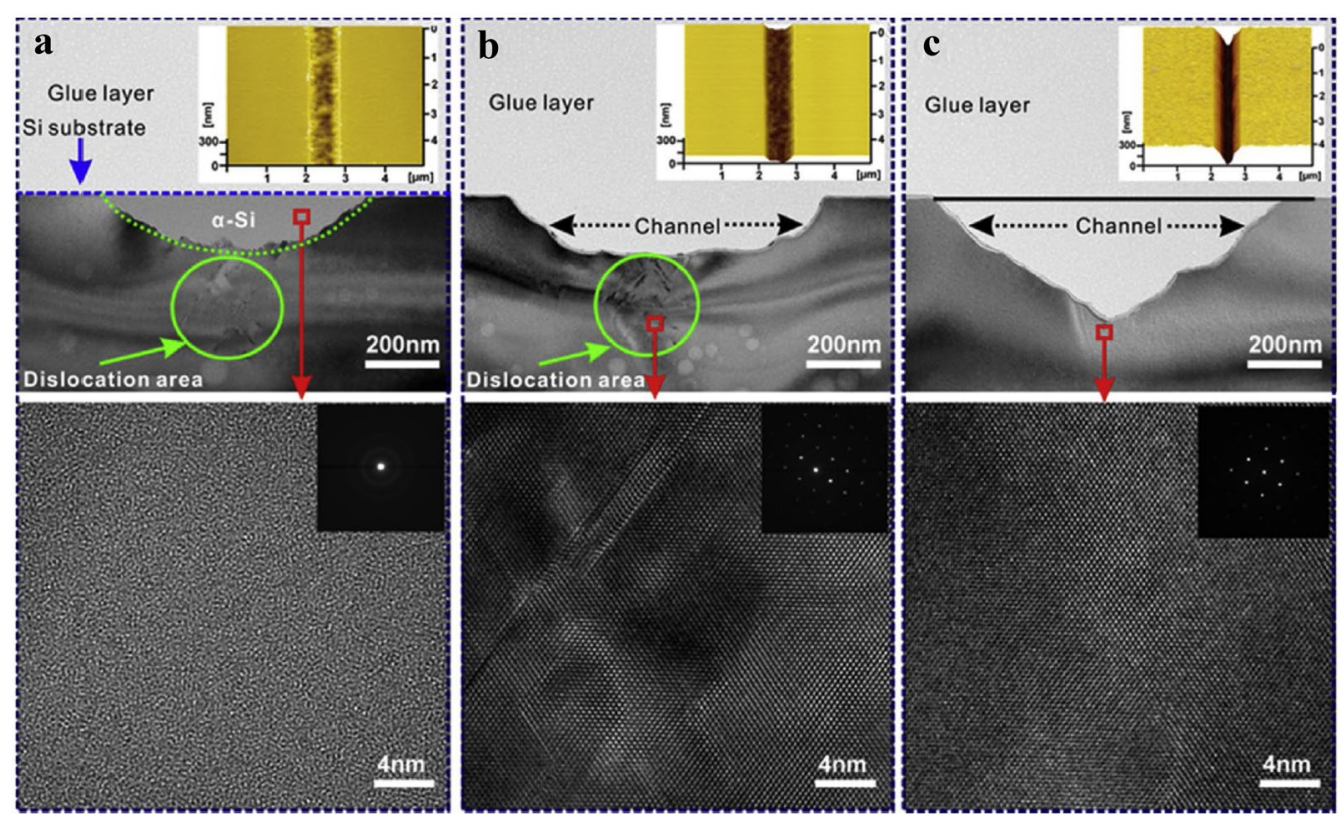

Figure $14 \mathrm{HTEM}$ detection on cross section of scratched regions before and after being etched by $\mathrm{HF}$ and $\mathrm{HF} / \mathrm{HNO}_{3}$ mixture [69] 


\subsection{Effects of Normal Load and Etching Time on Friction-Induced Selective Etching}

Normal load in scratching and etching time were demonstrated to show a significant effect on the formation of nanostructures by friction-induced selective etching. It is found that fabrication width was effectively controlled by the normal load, while fabrication height almost remained to stable after the scratches produced under contact pressure beyond $6.3 \mathrm{GPa}$ were etched by $\mathrm{KOH}$ solution $[27,56]$. Zhou et al. also found that the fabrication height is irrelevant with normal load during friction-induced selective etching in TMAH solution as long as the mask was active [67]. The fabrication height was both closely related with etching time in the two anisotropic etchants, i.e., $\mathrm{KOH}$ and TMAH solution, and the difference in etching time-dependent selective etching was attributed to subsurface deformation induced by diamond tip with different curvature radii. As a result, through selecting suitable normal load and etching time, various nanostructures with any layout patterns were fabricated on silicon surface by friction-induced selective etching in $\mathrm{KOH}$ and TMAH solution (Figure 15).

In case of $\mathrm{HF} / \mathrm{HNO}_{3}$ mixture etching, the fabrication height/width was tightly relevant to normal load in scratching and the time for post etching (Figure 16). Specifically, when low normal load is used for scratching, selective etching phenomenon was hardly observed, which can be ascribed to elastic deformation under the pressure less than yield limit of monocrystalline Si $(\sim 7$ $\mathrm{GPa}$ ). Protrusive hillocks were formed from higher loadinduced scratch $(2 \mathrm{~m} \cdot \mathrm{N})$ after the etching, and it can be attributed that amorphous silicon can act a mask in HF/
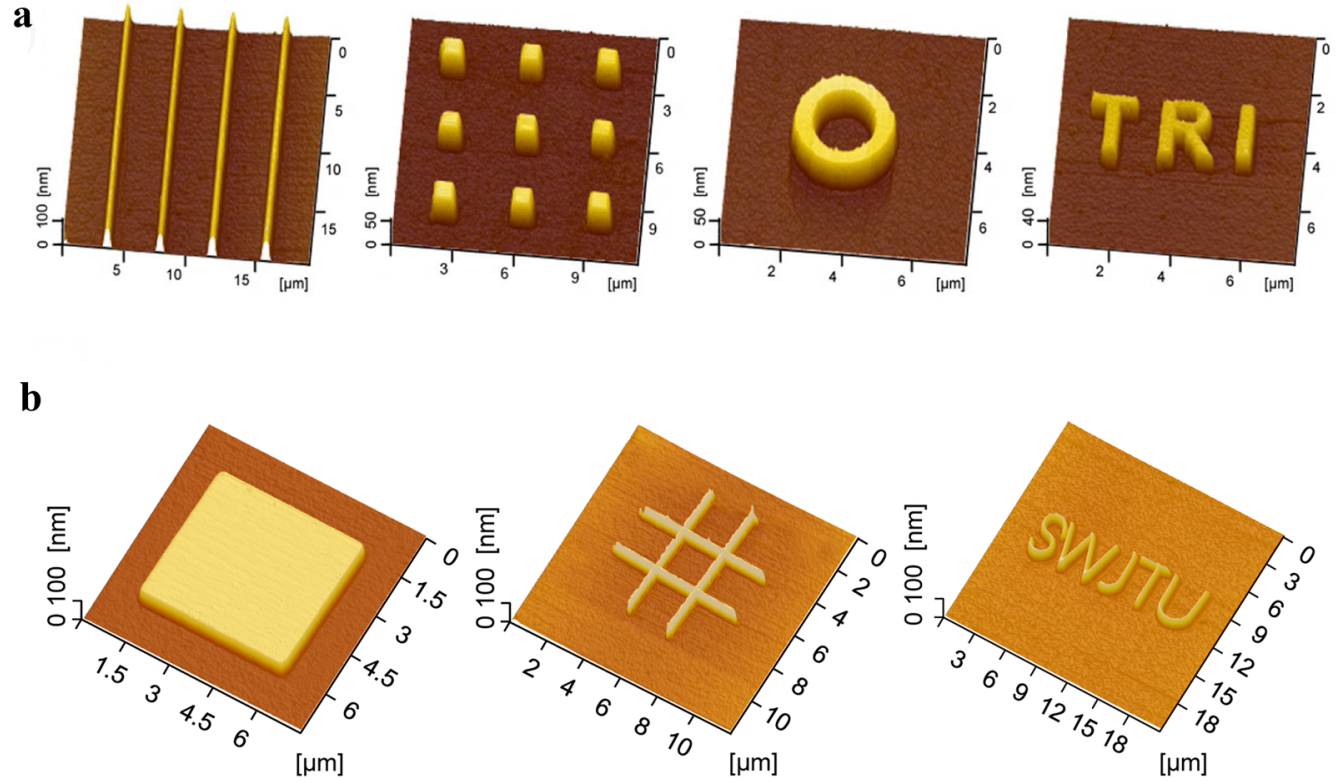

Figure 15 Fabrication of nanopatterns on Si surface through friction-induced selective etching in (a) $\mathrm{KOH}$ and (b) TMAH solution [27, 67]

a

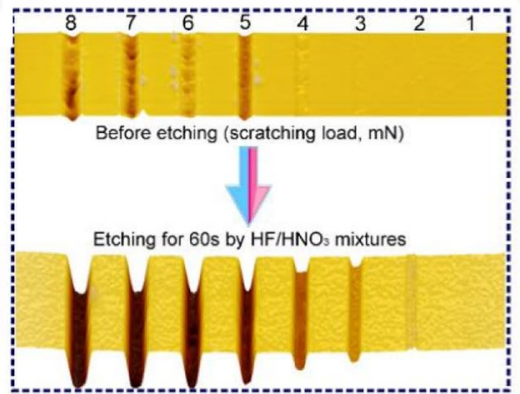

b

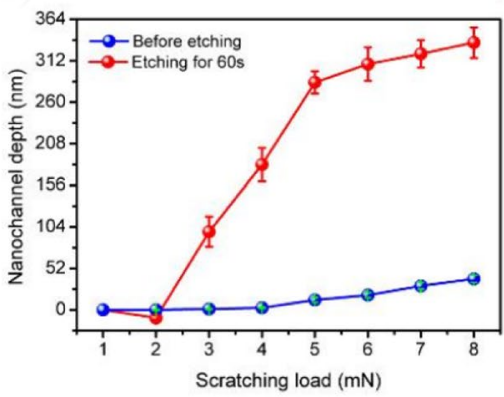

C

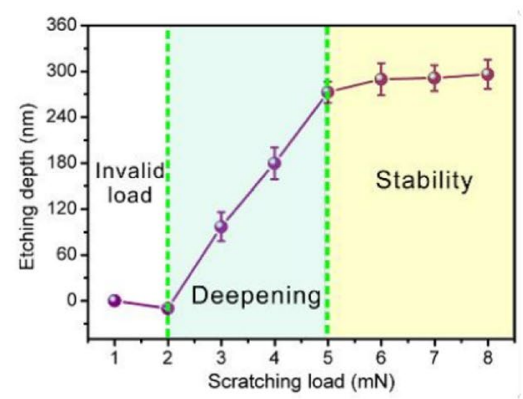

Figure 16 Normal load-dependent friction-induced selective etching in $\mathrm{HF} / \mathrm{HNO}_{3}$ mixture [69] 
$\mathrm{HNO}_{3}$ mixture etching [70]. However, scratches produced under excessive load can facilitate the etching in $\mathrm{HF} / \mathrm{HNO}_{3}$ mixture, resulting in the formation of deeper grooves, and the groove depth after the etching increased with the increase of applied load. Such results indicated that higher normal load can lead to more serious subsurface deformation, facilitating rapid selective etching. In addition, the fabrication depth stemming from the etching of higher-load scratches increased with etching time and then remained to be stable, and maximum depth was affected by volume ratio of $\mathrm{HF} / \mathrm{HNO}_{3}$ mixture.

\subsection{Effect of Temperature on Friction-Induced Selective Etching}

Aside from normal load and etching time, the temperature is an important parameter for establishing a controllable nanofabrication process by the selective etching. It is found that the fabrication height increased as the temperature raised before the collapse of the nanostructures during friction-induced selective etching in $\mathrm{KOH}$ solution (Figure 17a), and the selective etching rate was fitted well by Arrhenius equation (Figure 17b) [71]. Moreover, the etching process under different temperatures cannot introduce extra contamination based on XPS detections. It is also noted that surface properties, e.g., elastic modulus, hardness and contact angle, were obviously affected by etching temperature. Zhou et al found similar temperature-dependent selective etching performances in TMAH solution, and suggested that relatively low temperature, such as $25^{\circ} \mathrm{C}$, can be suitable for the realization of controllable nanofabrication due to the moderate etching rate. On the contrary, the fabrication depth decreased with etching temperature increased in $\mathrm{HF} / \mathrm{HNO}_{3}$ mixture [69].

As a result, even though the temperature increased etching selectivity in $\mathrm{KOH}$ solution, surface quality was reduced to some extent. Moreover, $\mathrm{HF} / \mathrm{HNO}_{3}$ mixture presented relatively poor selective etching characteristics and high surface roughness at high temperature. Hence, relatively low etching temperature was recommended for friction-induced selective etching nanofabrication.

\subsection{Nanoindentation-Induced Selective Etching}

It was well known that nanoindentation can cause subsurface deformation of silicon, and thus the indented regions were expected to act as a barrier layer to resist the etching. Accordingly, Jin et al. [72] proposed indentation-induced selective etching approach for the fabrication of silicon pyramidal nanotips (Figure 18). It was noted that indentation force and etching time had an obvious effect on fabrication process. Generally, under the same etching conditions, the height of newly formed pyramidal nanotip rises with the increase

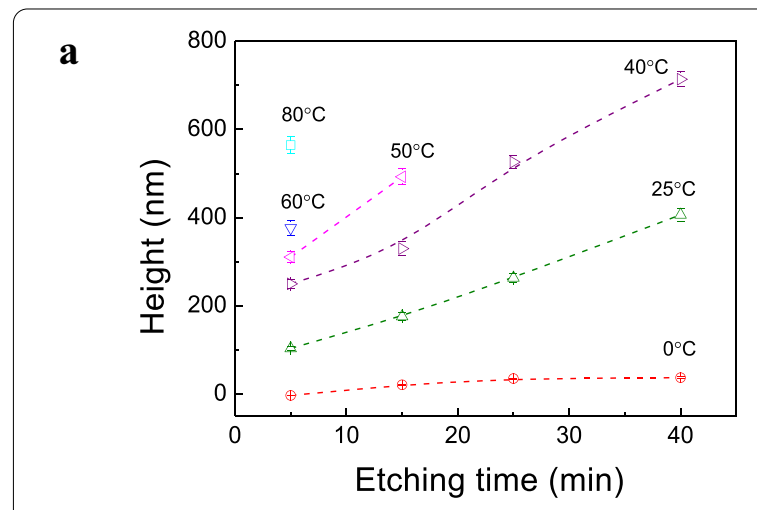

b

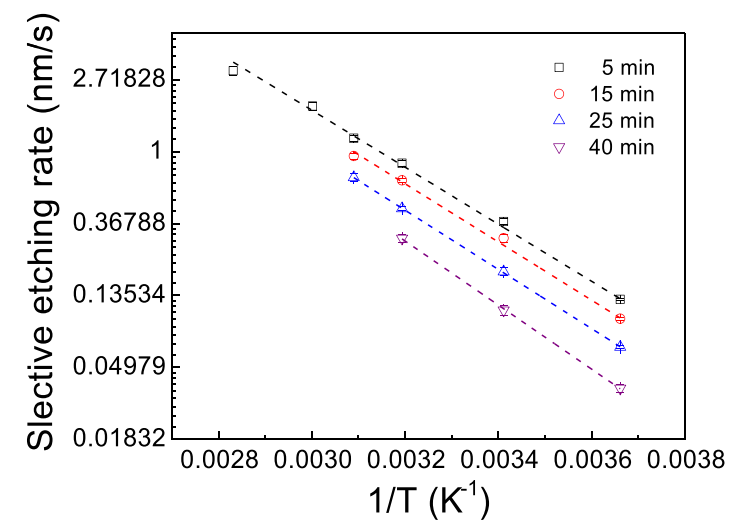

Figure 17 Temperature-dependent friction-induced selective etching on Si(100) surface [71]: (a) Correlation of fabrication height and etching time under various etching temperature during friction-induced selective etching in $\mathrm{KOH}$ solution, (b) The variation of the selective etching rate with reciprocal temperature $(1 / T)$

of indentation force, and low indentation force can facilitate the formation of sharp nanotips. The nanotips height and radius gradually increased and then decreased with etching time, and controllable fabrication was realized at 5-20 min. Further analysis indicated the formation of pyramidal tips is ascribed to anisotropic etching of silicon and etching stop of (111) crystal planes in $\mathrm{KOH}$ solution, and the mechanism was further verified by the fabricated nanostructures on $\mathrm{Si}(110)$ and $\mathrm{Si}(111)$ surfaces.

\section{Defect-free Nanofabrication by Friction-Induced Selective Etching on Silicon}

Crystal defects induced in fabrication process can degrade, to some extent, optical and electrical performances of Si-based devices, limiting their lifetime and reliability [64]. Specifically, defect-rich surfaces can cause rapid recombination of electron-hole pairs which decreases average carrier lifetime, and increase current leakage that may amplify noise [73]. In order to obtain defect-free Si nanostructures, it is necessary to optimize fabrication process of friction-induced selective etching. 

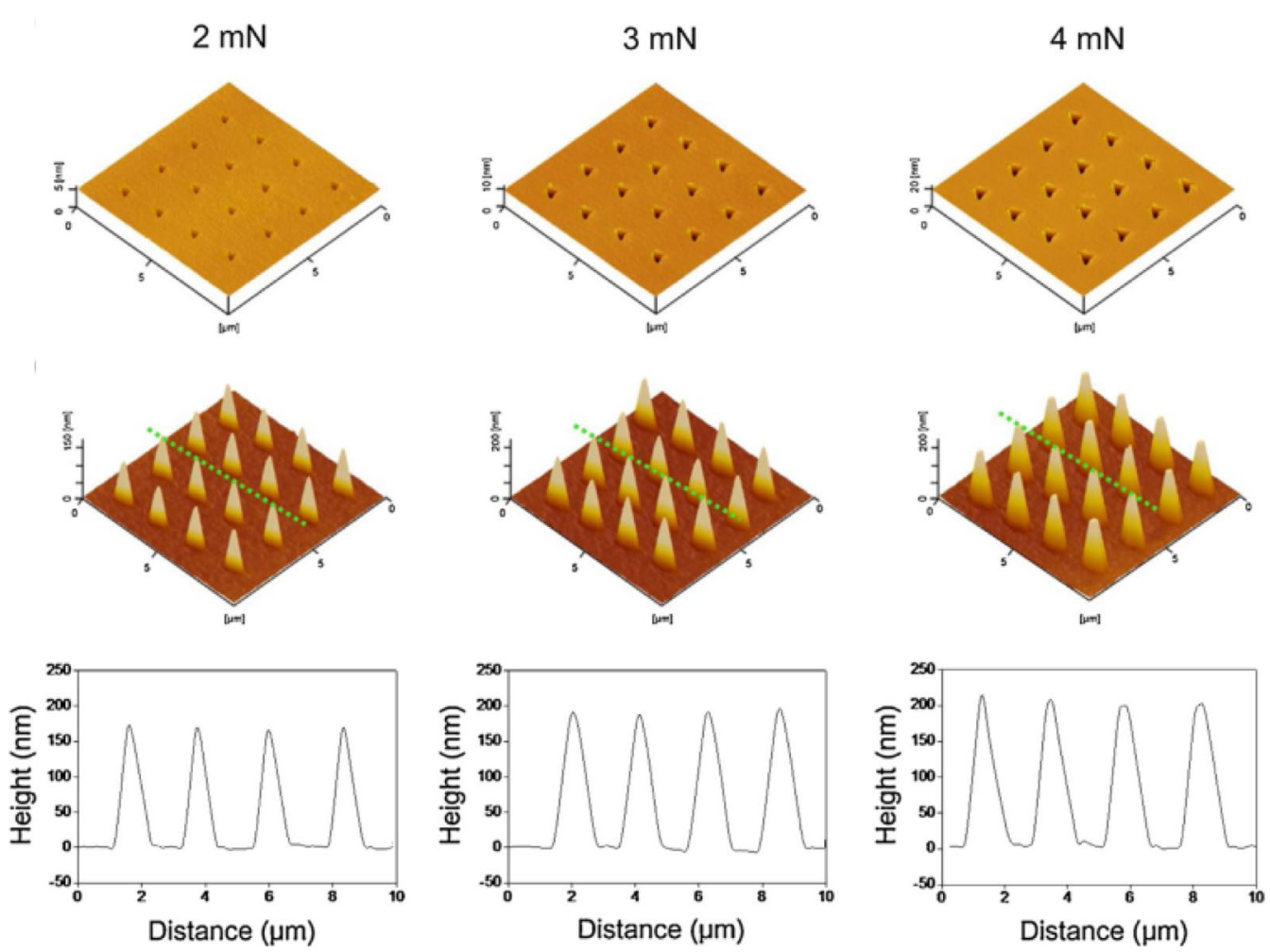

Figure 18 Fabrication of pyramid tip arrays by indentation-induced selective etching on silicon surface [72]: (a) \& (b) AFM images of indented regions produced under 2, 3, and $4 \mathrm{~m} \cdot \mathrm{N}$ before and after the etching in $\mathrm{KOH}$ solution for $10 \mathrm{~min}$, (c) cross-sectional profiles of fabricated tips were obtained from the dotted lines marked in (b)

\subsection{Tribochemistry-Induced Selective Etching}

Figure 19 displays the process of tribochemistryinduced selective etching for fabricating nondestructive nanostructures [74, 75]. Firstly, using an $\mathrm{AFM} \mathrm{SiO}_{2}$ tip, native oxidation layer on silicon substrate surface was removed by reciprocating scratching, where material removal was through tribochemistry reaction at contact interface. Since contact pressure between tip and sample during scratching process was less than yield limit of monocrystalline Si ( 7 GPa), no lattice damage occurred in scratched regions. Then, the scratched surface was immersed in $\mathrm{KOH}$ solution. Since native oxidization layer can act a mask against $\mathrm{KOH}$ etching, deeper nano-trench was produced by subsequent selective etching. Moreover, it was demonstrated that the proposed method can be realized at common humidity condition, and one scanning cycle was enough to remove $\mathrm{SiO}_{x}$ mask in area-scanning mode. HRTEM observations further revealed that the fabricated nanostructures were defect-free. The proposed approach was proven to be feasible for the fabrication of various nondestructive nanostructures, including nanotrenches array and multilayered pits.
Wang et al. [76] developed a UV/ozone-assisted tribochemistry-induced selective etching approach for the fabrication on silicon surface. UV/ozone system was used for the preparation of $\mathrm{SiO}_{x}$ film on surface, and the oxidation film was demonstrated to be more beneficial for subsequent fabrication in contrast with dry, chemi$\mathrm{cal}$, and thermal plasma-assisted oxidation. Formation mechanism of the oxidation film may be attributed to photo-oxidation reaction in the system, which mainly included two processes, i.e., organic decomposition and oxide densification [77]. Similarly, the oxidation film in the target area was removed to expose surface $\mathrm{Si}$ atom through tribochemistry reaction using an AFM $\mathrm{SiO}_{2}$ tip. Because of the difference in etching rate between oxidation film and exposed silicon regions, deeper nanogroove was formed by subsequent $\mathrm{KOH}$ solution etching, and fabrication depth was found to be dependent dramatically on oxidation time and etching time.

In addition, a simple method, i.e., site-controlled formation and removal of local oxide mask, was also developed to fabricate nondestructive nanostructures [20]. In this approach, a conductive AFM was firstly used for performing local anodic oxidation (LAO) on $\mathrm{H}$-passivation silicon surface, followed by immersing in TMAH solution 


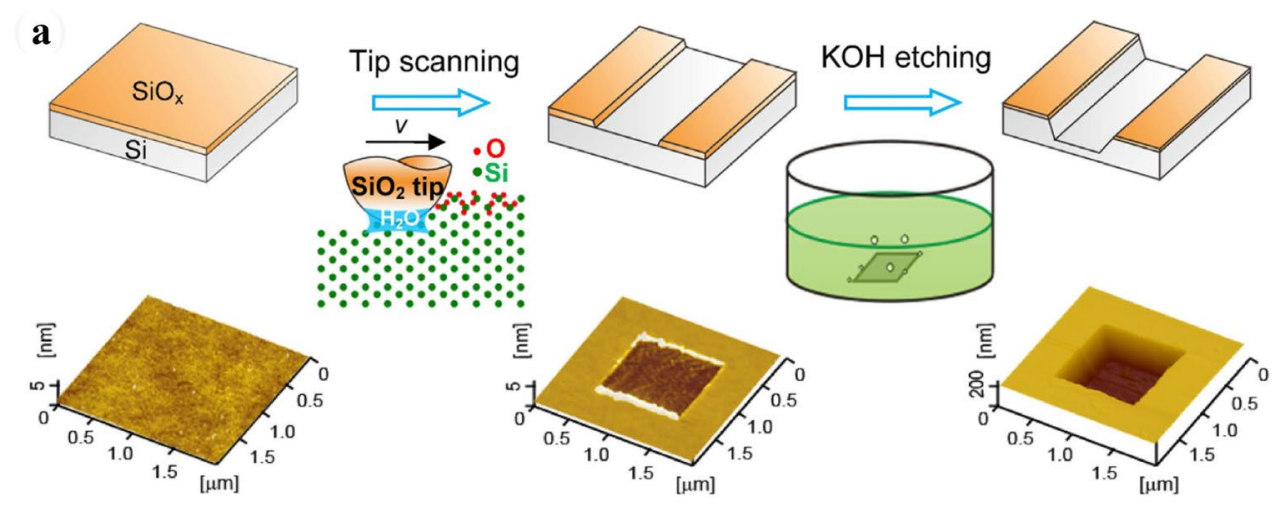

b

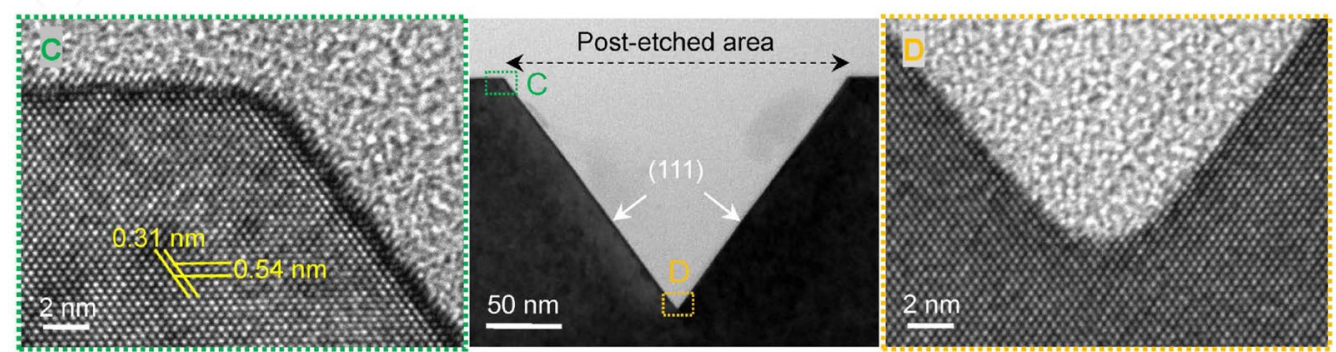

Figure 19 Defect-free nanofabrication by tribochemistry-induced selective etching [75]: (a) Fabrication process of tribochemistry-induced selective etching on silicon surface, (b) HRTEM observation of a defect-free groove with two magnified images on side panels

for selective etching, and then the fabricated nanostructures were dipped in HF solution to remove the oxidation layer. Through further verifying by CAFM detection and HRTEM observation, nanostructures produced by the approach were proven to be of perfect crystal lattice [20, $78]$.

\subsection{Friction-Induced Selective Etching of $\mathrm{Si}_{3} \mathrm{~N}_{4}$ Mask}

Figure 20 illustrates a nondestructive nanofabrication process on silicon surface through friction-induced selective etching of $\mathrm{Si}_{3} \mathrm{~N}_{4}$ mask [79]. The whole process mainly consists of four steps. Firstly, a diamond tip was used for scratching $\mathrm{Si}$ surface coated with $\mathrm{Si}_{3} \mathrm{~N}_{4}$ film. Secondly, the scratched sample was immersed in HF solution for selective etching the scratched $\mathrm{Si}_{3} \mathrm{~N}_{4}$ film until Si substrate was exposed. Here the scratched $\mathrm{Si}_{3} \mathrm{~N}_{4}$ film can be etched off faster than the intact film around the scratch. Then, the HF-etched $\mathrm{Si} / \mathrm{Si}_{3} \mathrm{~N}_{4}$ sample was dipped in $\mathrm{KOH}$ solution for further selective etching. Since the residual $\mathrm{Si}_{3} \mathrm{~N}_{4}$ film on non-scratched regions can act a mask to resist the etching, exposed $\mathrm{Si}$ on the bottom of the scratch was selectively etched in $\mathrm{KOH}$ solution and thereby form groove structure. Finally, the residual $\mathrm{Si}_{3} \mathrm{~N}_{4}$ film was removed completely by HF solution. Further analysis speculated that microcracks induced by the scratching can accelerate the diffusion of HF solution, promoting the etching of the scratched $\mathrm{Si} / \mathrm{Si}_{3} \mathrm{~N}_{4}$ surface. In contrast with traditional frictioninduced selective etching (Figure 18b), this method (Figure 18a) can fabricate forming nanostructures with lesser damage and deeper depths.

\section{Friction-Induced Selective Etching on Quartz, GaAs and Glass Surfaces}

Song et al. [80, 81] used friction-induced selective etching to fabricate nanostructures on quartz surface. Using an AFM diamond tip ( $R \approx 350 \mathrm{~nm}$ ), a scratched area without obvious wear was produced on quartz under area-scanning mode below the critical contact pressure of $5.1 \mathrm{GPa}$. The area was found to promote $\mathrm{KOH}$ solution etching, resulting in the formation of surface nanostructures. Through systematically investigating the effects of scan parameters and etching temperature on the formation of nanostructures, it was found that the etching thickness rose with the increase of the scan load and the number of scan cycles, but decreased with the scan speed. Furthermore, the rise of etching temperature can improve fabrication efficiency but hardly change etching thickness. However, the wear of quartz under higher normal load did not help to increase the etching thickness. HRTEM detections revealed no visible deformation under the scratch mask before and after being etched by 
a
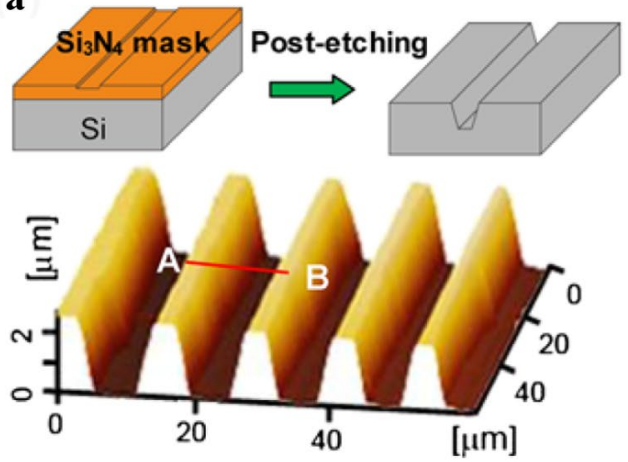

B
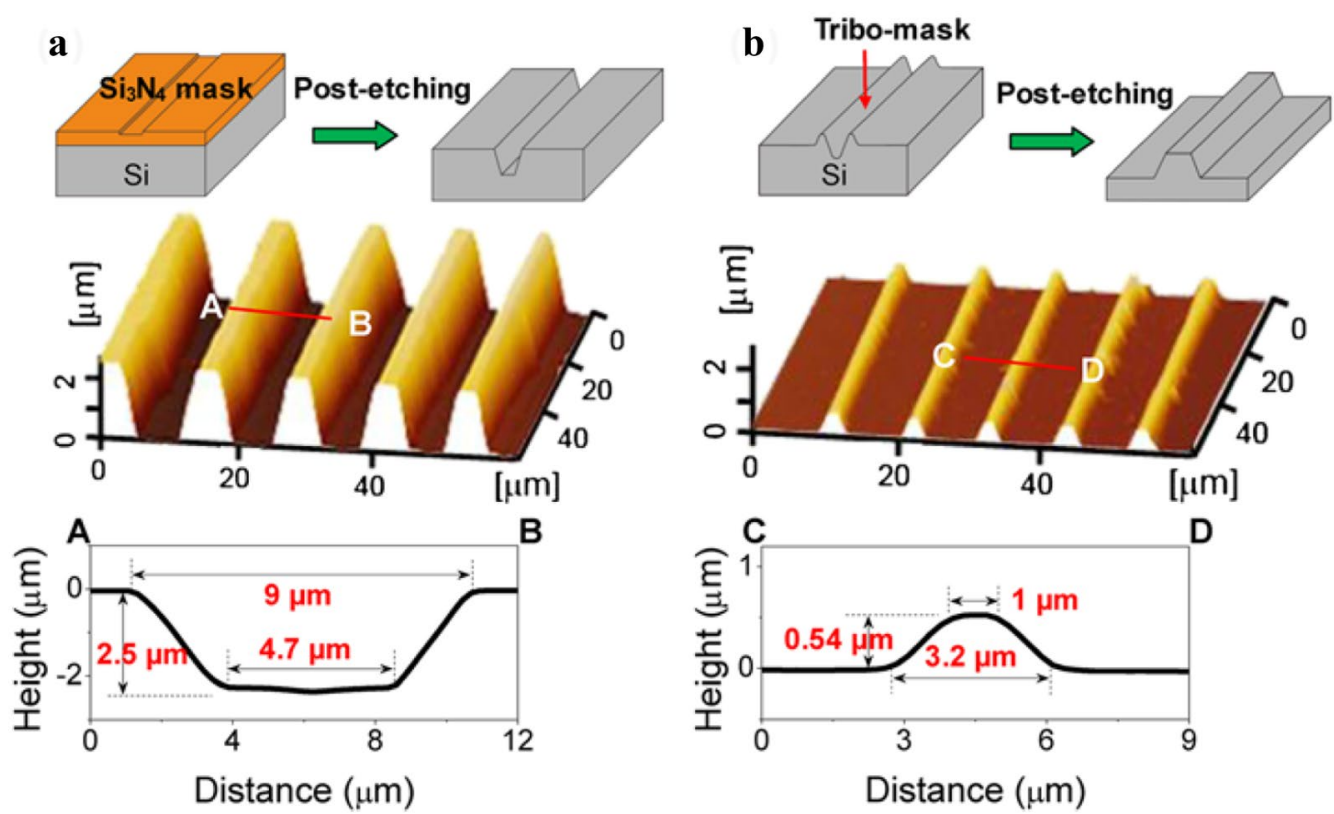

D

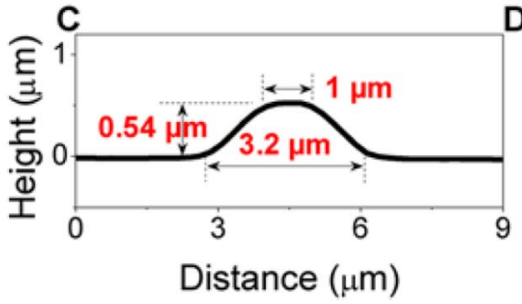

Figure 20 Fabrication of line-array patterns by friction-induced selective etching of $\mathrm{Si}_{3} \mathrm{~N}_{4}$ mask and traditional friction-induced selective etching [79]: (a) Friction-induced selective etching of $\mathrm{Si}_{3} \mathrm{~N}_{4}$ mask. Line-array pattern with $2.5 \mu \mathrm{m}$ in depth was fabricated by the scratching on $\mathrm{Si}_{3} \mathrm{~N}_{4}$ mask under $F_{n}=100 \mathrm{~m} \cdot \mathrm{N}$, post-etching in $\mathrm{HF}$ solution for $30 \mathrm{~min}$, and $\mathrm{KOH}$ solution for $4 \mathrm{~h}$ in sequence, (b) Traditional friction-induced selective etching (Line-array pattern with $0.54 \mu \mathrm{m}$ in height fabricated by scratching under $F_{\mathrm{n}}=70 \mathrm{mN}$ and post-etching in $\mathrm{KOH}$ solution for $1 \mathrm{~h}$ )

$\mathrm{KOH}$ solution. By depositing the gold film with a thickness of $\sim 300 \mathrm{~nm}$ on the half of amorphous $\mathrm{SiO}_{2}$ and crystal quartz surfaces, it was found that there was no etching difference between the covered and exposed surfaces on the crystal quartz surface after the etching by $\mathrm{KOH}$ solution and the removal of the gold layers, while a distinct step was formed on the amorphous $\mathrm{SiO}_{2}$ surface. Therefore, fabrication mechanism could be attributed to the selective etching of friction-induced amorphous layer on quartz surface, while lattice distortion provided little contribution for the selective etching. With the proposed etching, various nanostructures including slopes, hierarchical stages and chessboard-like patterns can be fabricated on quartz surface (Figure 21).

Combing diamond tip scratching and chemical etching in a mixture of sulfuric acid solution (volume ratio $\left.\mathrm{H}_{2} \mathrm{SO}_{4}: \mathrm{H}_{2} \mathrm{O}_{2}: \mathrm{H}_{2} \mathrm{O}=1: 0.5: 100\right)$, friction-induced selective etching was realized to produce hillock micro/nanostructures on GaAs surface (Figure 22a) [82]. The height of protrusive structures increased with the rise of normal
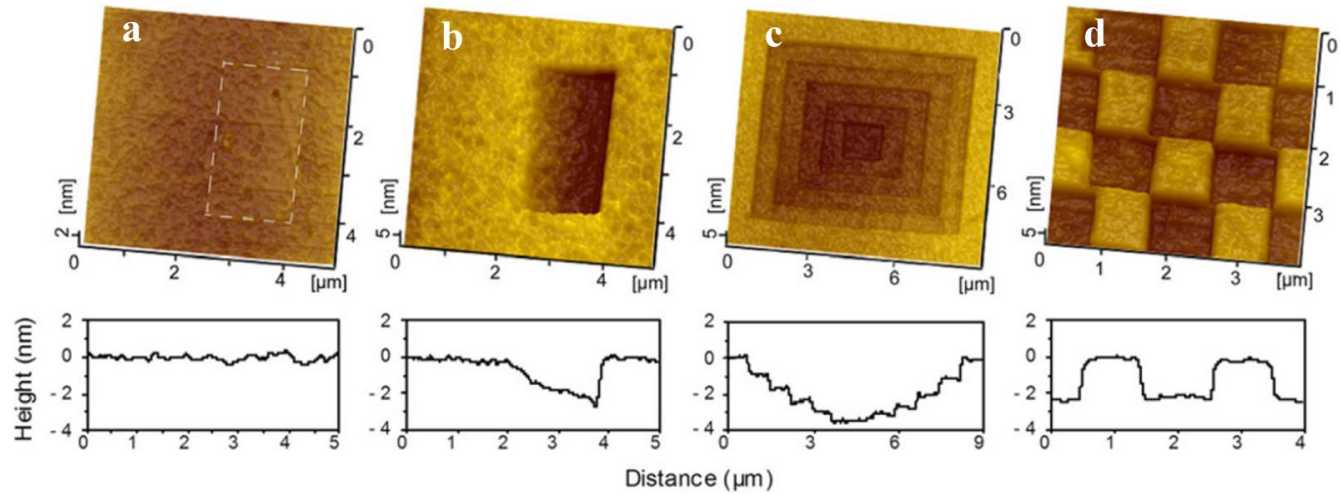

Figure 21 Friction-induced selective etching on quartz surface [80]: (a) A wearless area produced on quartz surface, (b)-(d) An inclined surface, hierarchical stages, and chessboard-like patterns through the proposed etching 

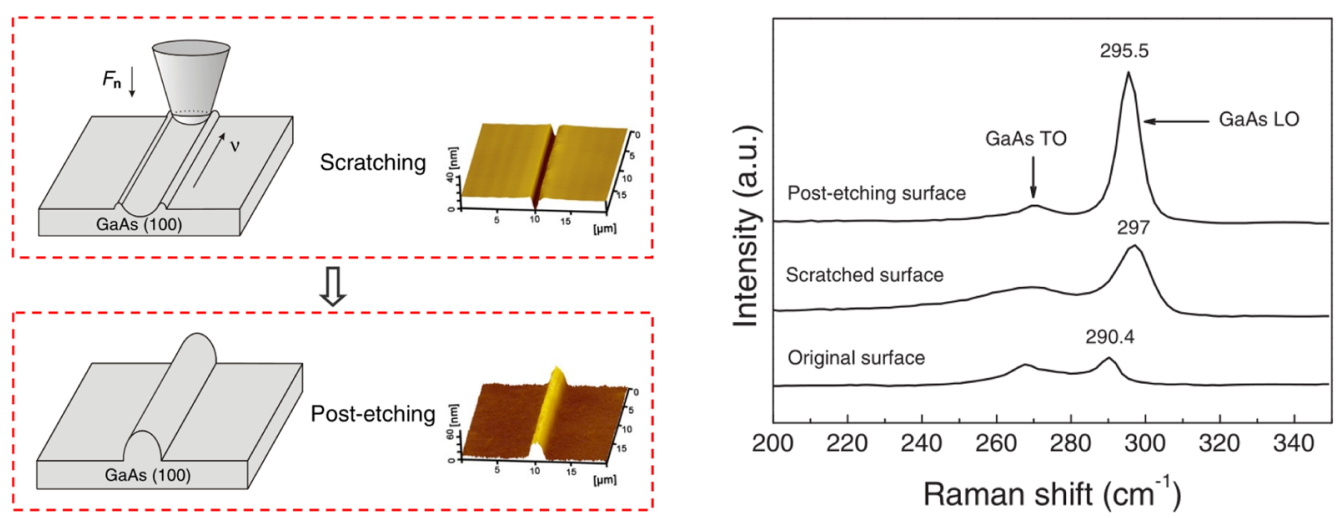

Figure 22 Friction-induced selective etching on GaAs surface [82]: (a) Schematic diagram and AFM images showing the fabrication process, (b) Raman detection on original GaAs surface, scratched surface, and post-etching surface (scratched surface after etching)

load or etching time. X-ray photoelectron spectroscopy detection confirmed that only slight tribochemical oxidation occurred on GaAs surface during the scratching (Figure 22b). Raman spectra on scratched GaAs surface suggested that longitudinal optical (LO) peak became wider and positive frequency shift was observed (Figure 22c), which can be attributed to structure disorder of GaAs lattice [83]. In view of this, one can suggest that residual compressive stress and lattice densification induced by the scratching may hinder the attack from the etchant, and hence the etching rate of the scratched region was slowed down. As a result, the difference of etching rate between untreated and scratched region resulted in the generation of hillock structures on GaAs surface in-situ from the scratched area. Through a homemade multi-probe instrument, the capability of this fabrication method was demonstrated by producing various nanostructures on GaAs surface, such as larger-scale linear array, intersecting parallel, surface mesas, and special letters.

Moreover, combing AFM diamond tip scratching and chemical etching in HF solution, friction-induced selective etching method was realized to fabricate hillock nanostructures on glass surface (atom concentration ratio $\mathrm{Al}: \mathrm{Na}: \mathrm{Si}=0.23: 0.07: 1$ ) [84]. The fabrication height increased as the scan load or the number of scan cycles increased. Further analysis based on X-ray photoelectron spectroscopy suggested that a densified layer would be generated at scanned regions because the angle of $\mathrm{Si}-\mathrm{O}-$ $\mathrm{Si}$ in glass decreased and the structures of glass was compressed [85]. The denser $\mathrm{AlF}_{3}$ layer can be formed in HF solution on scanned regions, and acts as a mask to prevent the etching from further etching by HF etchant compared to original glass surface [86], resulting in the formation of protrusive nanostructures. Moreover, it was demonstrated that the increase of scan load was beneficial to the formation of $\mathrm{AlF}_{3}$. By programming the loading mode and scanning traces, various nanostructures, such as slope, hierarchical steps, and designed patterns, can be produced by the proposed etching on glass surface (Figure 23).

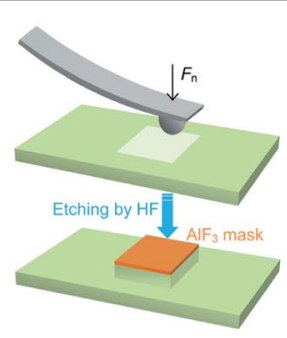

a

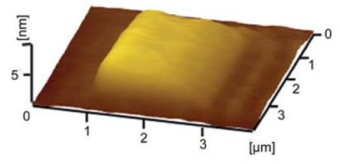

b

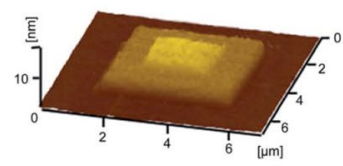

c

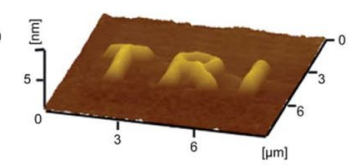

d

Figure 23 Friction-induced selective etching on glass surface [84]: (a) schematic diagram showing the fabrication process, (b)-(d) an inclined surface, hierarchical stages, and "TRI" pattern fabricated on glass surface 


\section{Rapid Nanofabrication by Friction-Induced Selective Etching}

The results shown in Section 3.3 demonstrated that high etching temperature was not ideal for improving fabrication efficiency because it can degrade surface properties. Similarly, to change the concentration of etching etchants seems to play limited role in promoting the nanofabrication efficiency by friction-induced selective etching. Accordingly, some researches were dedicated to exploring rapid fabrication approach based on friction-induced selective etching.

UV/ozone system was also introduced into frictioninduced selective etching on GaAs surface for improving the selectivity and reducing surface roughness [87]. It was found that the height of fabricated protrusive hillocks in UV/ozone-assisted system was obvious higher than that of traditional chemical etching within same etching time. Through systematically investigating fabrication parameters dependence of hillock height, it is found that the fabrication height firstly increased linearly and then decreased with etching time, and higher normal load in scratching can result in higher fabrication height. Further analysis suggested the rapid selective etching was attributed to photo-chemical etching of scratched GaAs surface. Specifically, less UV light was absorbed at the side of the grooves due to waveguide effects [88], resulting in a relatively dark region. For n-type materials, photoelectrons transferred to the relatively dark region, where the photo-electrons were consumed in the reduction of $\mathrm{HOGaAsOH}$, and hence the etching rate of scratched regions was lowered. Such a mechanism was also applied to indentation-induced selective etching. By programming tip scratching traces, various patterns with any layout and high-quality optical structures, such as circular gratings, diffraction gratings and pyramid-shaped array, were rapidly realized on GaAs surface (Figure 24).

Electrochemistry-assisted selective etching can also facilitate the rapid nanofabrication, which was developed to fabricate non-defective microstructures on silicon surface [89]. HRTEM observations indicated that both crystal distortion and amorphous layer in scratched regions can be etched rapidly whether the etching process was applied with positive or negative voltage. By investigating the effects of fabrication parameters, i.e., applied voltage, normal load and etching time, and substrate parameters including resistivity and crystal orientation on silicon microstructures, they found that all parameters except for the resistivity show significant effects on the depth and width of fabricated structures. Moreover, when applied voltage polarity was changed, the fabricated groove structures exhibited different cross-sectional profiles. Further analysis deduced that when the applied voltage was positive voltage, the curved structure would result in the enhancement of electrical filed at the groove area and hence many holes $\left(\mathrm{h}^{+}\right)$were accumulated around these regions, facilitating rapid etching of scratched regions (Figure 25a) [90, 91]. When the scratched silicon surface was etched by negative voltage, the $\mathrm{h}^{+}$inside the silicon and injected by the power source move toward the back side of the silicon (Figure 25b) [92]. Correspondingly,
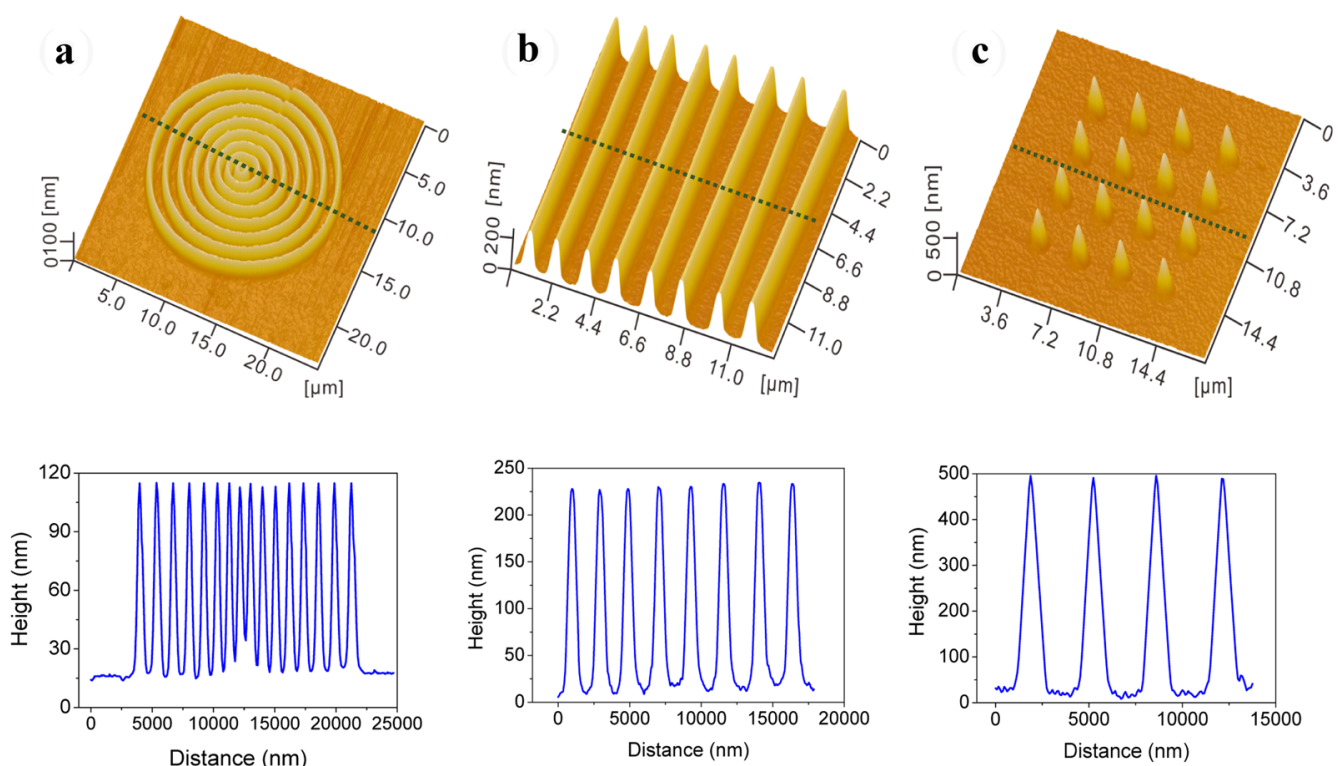

Figure 24 UV/Ozone-assisted fabrication of optical structures on GaAs surface [87]: (a) circular grating, (b) diffraction grating, and (c) pyramid-shaped array (All profiles at the bottom were obtained from the corresponding dotted lines on the upper AFM images) 


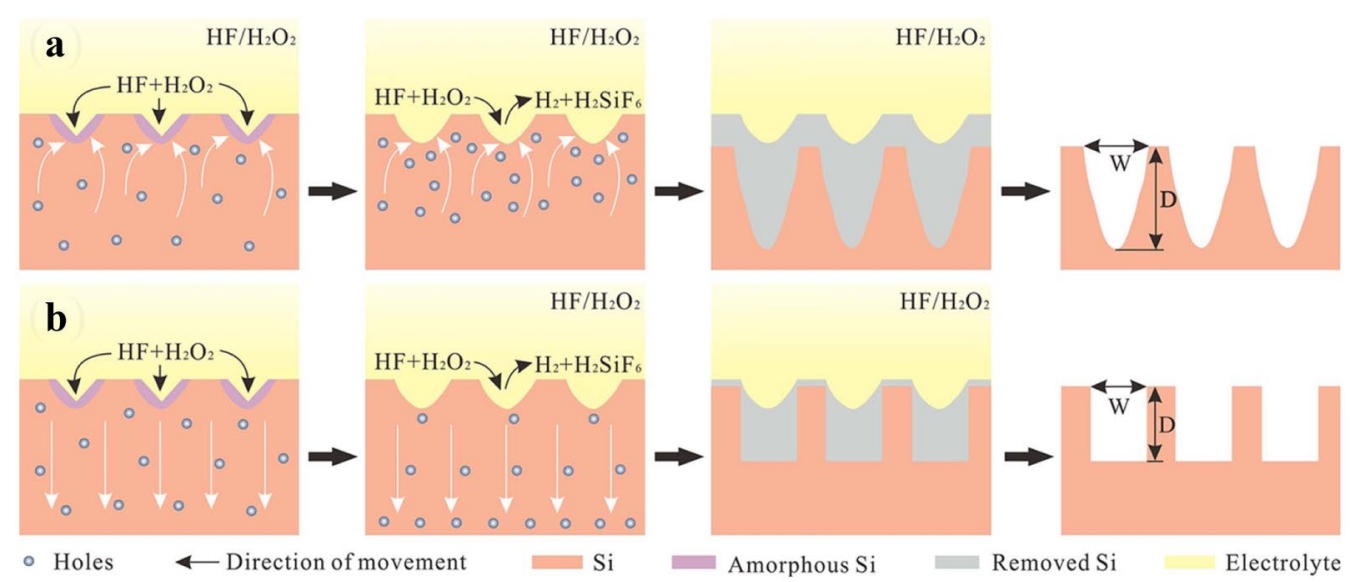

Figure 25 Etching mechanism of scratched silicon surface when the etching process applied with (a) positive voltage and (b) negative voltage in $\mathrm{HF} / \mathrm{H}_{2} \mathrm{O}_{2}$ solution [89]

the concentration of $\mathrm{h}^{+}$varied with the depth, forming a concentration gradient difference. The concentration of $\mathrm{h}^{+}$on silicon surface is the lowest, resulting in the generation of deeper groove structures on scratched silicon surface.

In addition, Wu et al. [93] developed a rapid fabrication method for fabricating nondestructive structures through site-controlled formation of friction-induced hillocks followed by selective etching in $\mathrm{HF} / \mathrm{HNO}_{3}$ mixture. In this study, it was found that friction-induced hillocks can act as a mask against the mixtures etching, and the fabrication process was significantly affected by volume ratio of $\mathrm{HF} / \mathrm{HNO}_{3}$ mixture, sliding cycle, normal load, and etching time. Further analysis suggested that lower average dangling bond density in scanned regions may retard the breakup of $\mathrm{Si}-\mathrm{H}$ bonds and polarized $\mathrm{Si} \mathrm{Si}$ backbonds [27], leading to lower dissolution rate in HF/ $\mathrm{HNO}_{3}$ mixture compared to original $\mathrm{Si}$ surface. Crosssectional HRTEM observations revealed defect-free nanostructures fabricated by the anisotropic etching. Also, several nondestructive nanostructures with different patterns were realized on Si surfaces by programming tip scanning traces. The proposed method is promising for the fabrication of controllable nano-sized functional devices by $\mathrm{HF} / \mathrm{HNO}_{3}$ mixture.

\section{Applications of Friction-Induced Nanofabrication}

Based on friction-induced fabrication fundamentals, $\mathrm{Wu}$ et al. [94] designed and fabricated a multi-probe microfabrication apparatus, which greatly improved the fabrication efficiency. The apparatus mainly consisted of actuating device, loading system and control system. Figure 26a illustrated main parts of the multi-probe micro-fabrication apparatus. With a motorized $X / Y$ linear stage, the scanning can be realized over a $50 \mathrm{~mm} \times 50 \mathrm{~mm}$ area with a maximum sliding speed of $10 \mathrm{~mm} / \mathrm{s}$. Moreover, a deformable parallelogram with two beams was applied to precisely control normal load in scratching. After performing indentation tests, fabricated indents array can be used to locate steel micro balls and thereby prepare multi-probe array (Figure 26b) [95]. Combining probe scanning and post etching in $\mathrm{KOH}$ solution, various micro-patterns including line arrays and ring-shaped structures were fabricated on $\mathrm{Si}(100)$ surface.

With the friction-induced selective etching, Si-based master gratings were produced for UV-assisted nanoimprint lithography [96]. Effects of surface cleaning method and diamond tip shape on the fabrication were systematically investigated to obtain high-quality grating structures. Compared to conventional cleaning method, magnetic stirring assistant cleaning was demonstrated to perform better for removing chemical reaction products and recommended for the production of $\mathrm{Si}$-based gratings via the selective etching. It was also noted that sharp and smooth diamond tip was more beneficial for fabricating high-quality gratings compared with blunt diamond tip. Moreover, the fabrication height/width and micromorphology of grating structures are strongly dependent on the normal load in scratching and the time for post etching, providing a guideline for controllable fabrication. Using UV nanoimprint lithography technology, the fabricated master grating structures were excellently replicated on polymer surface. Furthermore, it was demonstrated through in-situ characterization approach with SEM and AFM that the structural features of master grating were perfectly replicated, as shown in Figure 27. 


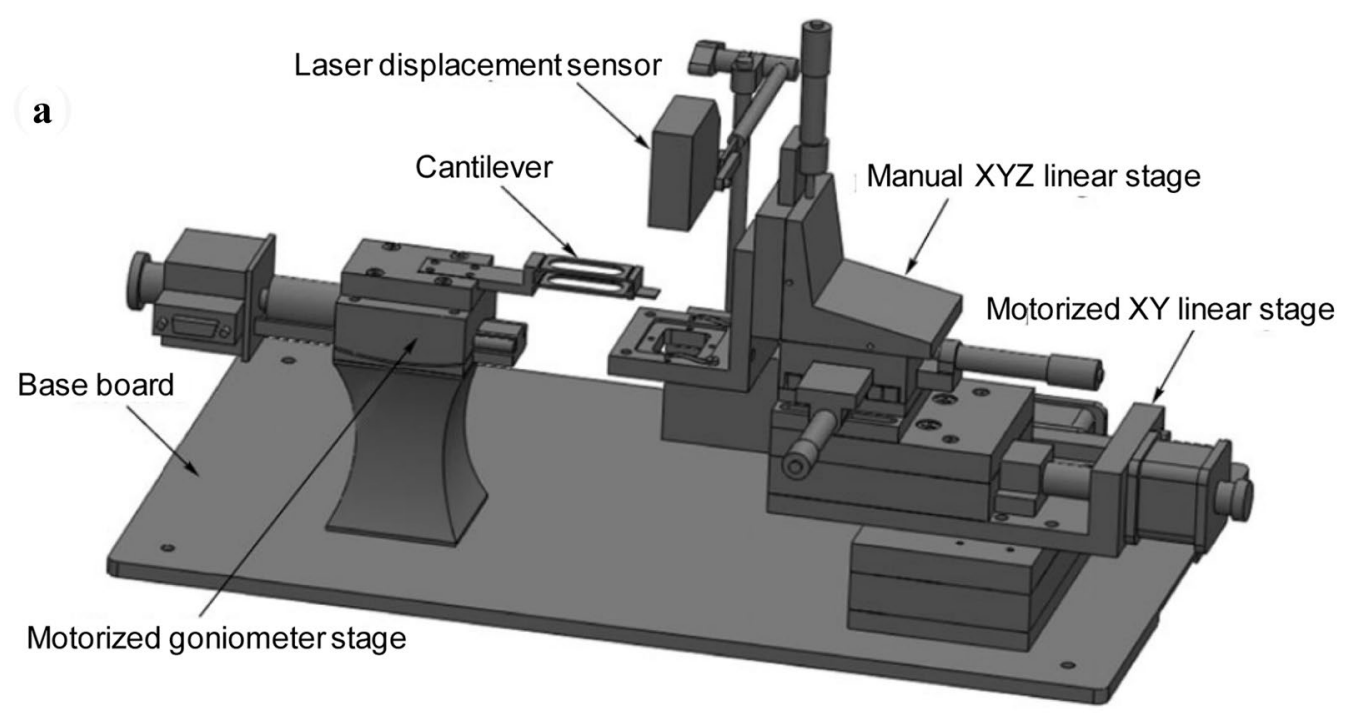

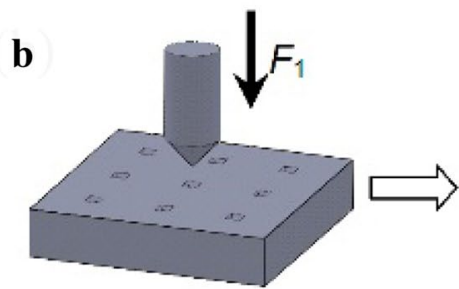

I

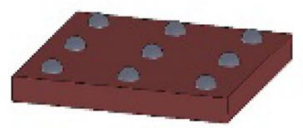

VI

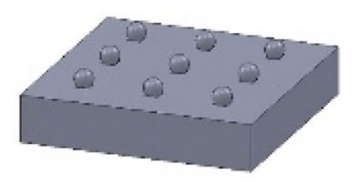

II
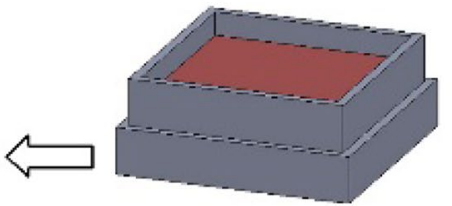

V

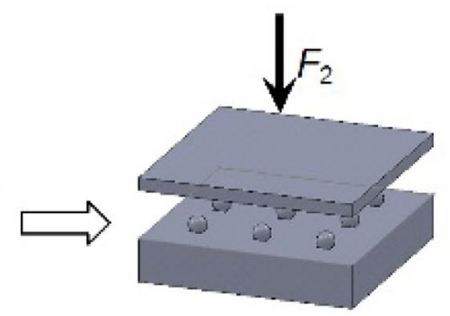

III

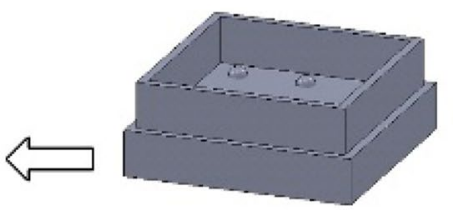

IV

Figure 26 Schematic illustration of (a) mechanical part of the multi-probe fabrication apparatus, and (b) the preparation of multi-probe array [94, 95]

Friction-induced selective etching provide great opportunities in realizing nanochannels [69, 97], which was widely used in fields covering microfluidics, chemistry, biology and so forth. Wang et al. [69] designed, fabricated and characterized a nanofluidic device based on frictioninduced selective etching. The device mainly consists of ports, seals, micro/nanochannels and substrate layers, as shown in Figure 28. The two reservoirs were produced by multi-probe micro-fabrication apparatus in Figure 26 on Si surface. The seals and microchannel layers were fabricated by filling liquid polydimethylsiloxane (PDMS) into a special mold, and dark-field fluorescent characterization showed that the interface was closely combined without distinct side leakage, demonstrating that the device had a good bonding quality (Figure 28e \& f). Electrical measurements in $1.0 \mathrm{mM} \mathrm{KCl}$ indicated that nanochannel current exhibited a good linear relationship with applied voltage, and decreased as a function of time, which can be attributed that the electrolyte at anodic side of the nanochannel interface was gradually depleted. Moreover, the depth and width of the fabricated channels are larger than Debye length of the electrolyte $(\sim 9.6 \mathrm{~nm})$, and hence the electrical double layers could not overlap in the nanochannels and affect electro-osmosis [98]. The 


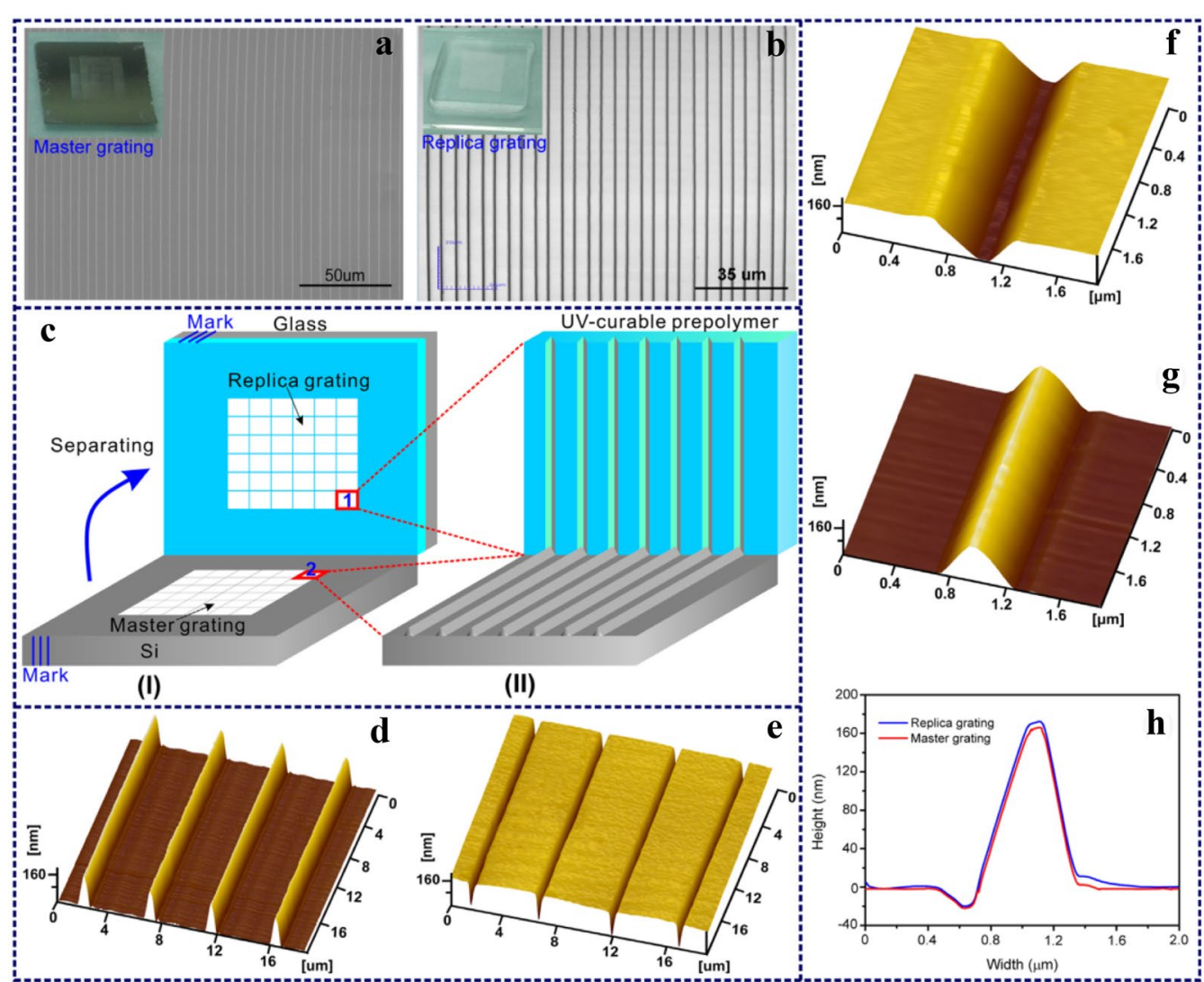

Figure 27 UV-assisted nanoimprint lithography of Si-based master grating structures fabricated by friction-induced selective etching [96]

above results indicated that the prepared nanochannels had an excellent performance and thereby can meet practice application needs for nanofluidic analysis.

\section{Conclusions and Perspective Remarks \\ 8.1 Conclusions}

This paper reviewed the fundamentals and applications of friction-induced nanofabrication, including frictioninduced selective etching. Firstly, direct nanofabrication was demonstrated by mechanical removal and frictioninduced hillocks. The generation process of frictioninduced hillocks on monocrystalline silicon surface was addressed regarding the influence of experimental conditions, including crystal planes, atmosphere, sliding velocity, etc, and the mechanism was further clarified as that the hillocks are dominated by amorphous silicon rather than oxides, which provides the fundamental for friction-induced selective etching. When the surface of monocrystalline silicon with scratches is etched by a specific solution, such as $\mathrm{KOH}$, TMAH or $\mathrm{HF} / \mathrm{HNO}_{3}$ solution, protrusive hillock or deeper groove will be produced; the mechanisms were addressed based on the mask effects of different lattice deformation and surface oxides for resisting or promoting the etching in a given enchant. With friction-induced etching, defect-free nanostructures were fabricated via controllable preparation and tribochemical removal of silicon oxide mask by tip scratching on silicon surface. Friction-induced selective etching on quartz, GaAs and glass was shown with the fabrication mechanisms. To improve the efficiency, rapid nanofabrication by friction-induced selective etching was developed with externally applied UV radiation system and electric field. Finally, the applications of friction-induced nanofabrication were demonstrated by the building of multi-probe apparatus for large-scale scratching, and the fabrication of nanoimprint templates and nanochannels.

As a newly developed SPL method, friction-induced nanofabrication has the merits of little pollution to environment, low processing damages, strong flexibility, and low cost. Without any template, the nanofabrication can be realized on silicon, GaAs, quartz, glass surfaces, and so on, and can find its broad applications in the processing of functionalized surface textures and micro/ nano devices, such as high-quality optical substrates and 

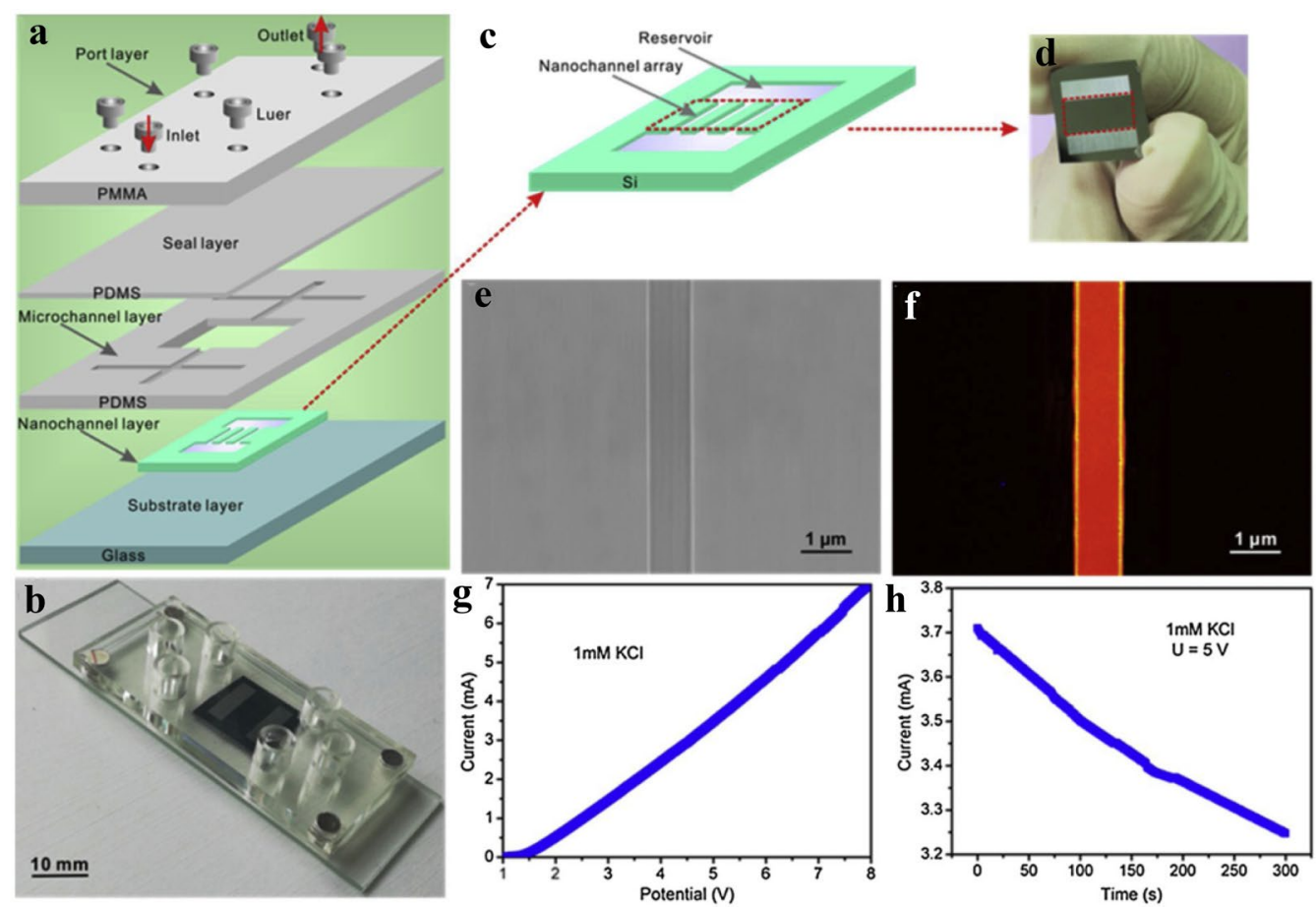

Figure 28 Design, fabrication, and characterization of a nanofluidic device based on friction-induced selective etching technique [69]

biochips. However, some remarks remain to be noted for improving the friction-induced fabrication.

\subsection{Perspective Remarks}

Although friction-induced nanofabrication technique has been investigated for years, most existing investigations were focused on fabrication mechanism. In order to advance the application of this technique in high performance devices and even in semiconductor industry, more studies still need to be conducted in the future.

\subsubsection{Generation Mechanism of Friction-Induced Hillocks}

To clarify the generation mechanism of friction-induced hillocks can not only enrich the theoretical knowledge of tribology, but also provide further scientific support for wet etching process. Previous work was focused on revealing the contribution of oxidation and mechanical interaction to the height of friction-induced hillocks on silicon surface, while specific formation mechanism was still not clearly understood up to now. Establishing a widely accepted formation mechanism for frictioninduced hillocks on various material surfaces is still to be done in the future. Moreover, uncovering the generation mechanism of friction-induced hillocks is more significant for the controllable fabrication with frictioninduced selective etching.

\subsubsection{Optimization for Aspect Ratio of Fabricated Structures}

Usually, friction-induced nanofabrication method can be employed for realizing structures with limited aspect ratio. Proper aspect ratio is required for the nanostructure to realize devices. For example, a small slope angle $\left(<20^{\circ}\right)$ is required for high quality blazed gratings, while high aspect ratio structures are significant for X-ray diffractive optics, such as zone plates [99]. Some strategies, such as reducing tip radius, applying an electric or magnetic field during scratching process to change the type of subsurface deformation, and friction-induced selfassembly film formation to improve anti-etching ability of scratched regions, may be attempted to improve aspect ratio of fabricated structures. Additionally, some methods, such as adding additives into wet etchants or employing alternative substrate (e.g., $\mathrm{Si}(110)$ ) to change the etching behavior, can be considerate to control the shape of fabricated structures. 


\subsubsection{Development of Large-Area and High-Precision Multi-Probe Nanofabrication Equipment}

Usually, a single probe was used to scratch sample surfaces for friction-induced nanofabrication, resulting in low fabrication efficiency. The lack of fabrication equipment with large-area and high-precision ability hindered seriously widespread application of this method, and the corresponding equipment is urgent to be developed. However, when scaling down fabrication dimensionality towards nanoscale, the design, fabrication, installation, inspection, and control of multi-probe equipment face huge challenges.

\subsubsection{Realization of Functional Surface and Structures Based on Friction-Induced Nanofabrication}

Friction-induced nanofabrication has been demonstrated to be a robust way for fabricating surface structures because of its high resolution, good flexibility, and cost-effective manner. The potential applications mainly include but not limited by the following aspects.

- Nanochannels for high-performance nanofluidic chips, including microreactors and surface enhanced Raman substrates (SERS).

- High-quality grating structures, such as blazed gratings, circular gratings and diffraction gratings, for high-performance photodetector or optoelectronic devices.

- Special structures, such as nanoline arrays, nanoholes, micro/nano pyramids and so forth, for lighttrapping devices.

- Patterned substrates for site-controlled growth of nanostructures by molecular beam epitaxy or chemical deposition.

\section{Acknowledgements}

The authors sincerely thanks to Mr. Lei Wu, Mr. Chengqiang Feng and Mr. Liang Deng of Southwest Jiaotong University for their formatting references, preparing figures and making corrections during manuscript preparation.

\section{Authors' Information \\ Bingjun Yu, born in 1981, is currently an associate professor at Southwest Jiaotong University (SWJTU), China. He received his PhD degree from SWITU in 2012. His current research focuses on scanning probe microscopy (SPM)- based nanofabrication, nanomanipulation and nanotribology. \\ Linmao Qian, born in 1971, is currently a professor at SWJTU, China. He received his PhD degree from Tsinghua University, China, in 1999. His current research focuses on ultra-precision surface manufacturing, SPM-based nano- fabrication, nanotribology and intelligent manufacturing.}

\section{Authors' Contributions}

BY optimized the presenting structure and wrote the manuscript; LQ designed the presenting structure and polished the writing of the manuscript. All authors read and approved the final manuscript.
Funding

Supported by National Natural Science Foundation of China (Grant Nos. 51775462, 51991373).

\section{Competing Interests}

The authors declare no competing financial interests.

Received: 5 November 2020 Revised: 4 February 2021 Accepted: 26 February 2021

Published online: 17 March 2021

\section{References}

[1] A Pimpin, W Srituravanich. Review on micro- and nanolithography techniques and their applications. Engineering Journal, 2012, 16(1): 37-56.

[2] H Phan, T Nguyen, T Dinh, et al. Robust free-standing nano-thin sic membranes enable direct photolithography for MEMS sensing applications. Advanced Engineering Materials, 2018, 20(1): 1700858.

[3] D Qin, Y Xia, G M Whitesides. Soft lithography for micro- and nanoscale patterning. Nature Protocols, 2010, 5: 491-502.

[4] SY Chou, C Keimel, J Gu. Ultrafast and direct imprint of nanostructures in silicon. Nature, 2002, 417(6891): 835-837.

[5] J Deng, J Yan, J Shao, et al. Electron beam lithography on uneven resist for uniform Bragg gratings in Ga-Sb based distributed-feedback laser. Microelectronic Engineering, 2018, 195: 32-35.

[6] L D Menard, J M Ramsey. Fabrication of sub-5 nm nanochannels in insulating substrates using focused ion beam milling. Nano Letters, 2011, 11: 512-517.

[7] $Y X u, N$ Matsumoto. Flexible and in situ fabrication of nanochannels with high aspect ratios and nanopillar arrays in fused silica substrates utilizing focused ion beam. RSC Advances, 2015, 5: 50638-50643.

[8] Y K Ryu Cho, C D Rawlings, H Wolf, et al. Sub-10 nanometer feature size in silicon using thermal scanning probe lithography. ACS Nano, 2017, 11(26): 11890-11897.

[9] S Chen, S Kim, W Chen, et al. Monolayer MoS2 nanoribbon transistors fabricated by scanning probe lithography. Nano Letters, 2019, 19(3): 2092-2098.

[10] Y Wang, X Hong, J Zeng, et al. AFM tip hammering nanolithography. Small, 2009, 5(4): 477-483.

[11] G Binnig, H Rohrer, Ch Gerber, et al. Surface studies by scanning tunneling microscopy. Physical Review Letters, 1982, 49(1): 31-35.

[12] A A Tseng. Tip-based nanofabrication: fundamentals and applications. Phoenix: Arizona State University, 2011.

[13] A P Malshe, K P Rajurkar, K R Virwani, et al. Tip-based nanomanufacturing by electrical, chemical, mechanical and thermal processes. CIRP Annals Manufacturing Technology, 2010, 59(2): 628-651.

[14] D M Eigler, E K Schweizer. Positioning single atoms with a scanning tunneling microscope. Nature, 1990, 344(6266): 524-526.

[15] F J Giessibl, C F Quate. Exploring the nanoworld with atomic force microscopy. Physics Today, 2006, 59(12): 44-50

[16] Y Sugimoto, M Abe, S Hirayama, et al. Atom inlays performed at room temperature using atomic force microscopy. Nature Materials, 2005, 4(2): 156-159.

[17] O Custance, R Perez, S Morita. Atomic force microscopy as a tool for atom manipulation. Nature Nanotechnology, 2009, 4(12): 803-810.

[18] H C Manoharan, C P Lutz, D M Eigler. Quantum mirages formed by coherent projection of electronic structure. Nature, 2000, 403(6769): 512-515.

[19] D Stiévenard, B Legrand. Silicon surface nano-oxidation using scanning probe microscopy. Progress in Surface Science, 2006, 81 (2): 112-140.

[20] G Guo, J Li, C Deng, et al. Nondestructive nanofabrication on monocrystalline silicon via site-controlled formation and removal of oxide mask. Applied Physics Express, 2018, 11(11): 116501.

[21] Y Li, B W Maynor, J Liu. Electrochemical AFM "Dip-Pen" nanolithography. Journal of the American Chemical Society, 2001, 123(9): 2105-2106

[22] R D Piner, J Zhu, F Xu, et al. "Dip-Pen" nanolithography. Science, 1999, 283(5402): 661-663.

[23] D Pires, J L Hedrick, A D Silva, et al. Nanoscale three-dimensional patterning of molecular resists by scanning probes. Science, 2010, 328(5979): $732-735$ 
[24] B Yu, H Dong, L Qian, et al. Friction-induced nanofabrication on monocrystalline silicon. Nanotechnology, 2009, 20(46): 465303.

[25] B Yu, L Qian, H Dong, et al. Friction-induced hillocks on monocrystalline silicon in atmosphere and in vacuum. Wear, 2010, 268(9): 1095-1102.

[26] BYu, X Li, H Dong, et al. Towards a deeper understanding of the formation of friction-induced hillocks on monocrystalline silicon. Journal of Physics D: Applied Physics, 2012, 45(14): 145301.

[27] J Guo, C Song, X Li, et al. Fabrication mechanism of friction-induced selective etching on Si (100) surface. Nanoscale Research Letters, 2012, 7(1): 152

[28] Y D Yan, Z J Hu, X S Zhao, et al. Top-down nanomechanical machining of three-dimensional nanostructures by atomic force microscopy. Small 2010, 6(6): 724-728

[29] J Deng, JY Dong, Paul Cohen. High rate 3D nanofabrication by AFMbased ultrasonic vibration assisted nanomachining. Procedia Manufacturing, 2016, 5: 1283-1294.

[30] U Kunze. Nanoscale devices fabricated by dynamic ploughing with an atomic force microscope. Superlattices and Microstructures, 2002, 31(1): 3-17.

[31] Y He, Y D Yan, Y Q Geng, et al. Fabrication of periodic nanostructures using dynamic plowing lithography with the tip of an atomic force microscope. Applied Surface Science, 2018, 427: 1076-1083.

[32] B Vasic, M Kratzer, A Matkovic, et al. Atomic force microscopy based manipulation of graphene using dynamic plowing lithography. Nanotechnology, 2013, 24: 015303.

[33] R Kaneko, T Miyamoto, Y Andoh, et al. Microwear. Thin Solid Films, 1996, 273(1): 105-111.

[34] E Hamada, R Kaneko. Micro-distortion of polymer surfaces by friction. Journal of Physical D: Applied Physics, 1992, 25: A53.

[35] R Kaneko, E Hamada. Microwear processes of polymer surfaces. Wear, 1993, 162-164: 370-377.

[36] J Yu, L Qian, B Yu, et al. Nanofretting behaviors of monocrystalline silicon (100) against diamond tips in atmosphere and vacuum. Wear, 2009, 267(1): 322-329.

[37] T Fang, C Weng, J Chang. Machining characterization of the nano-lithography process using atomic force microscopy. Nanotechnology, 2000, 11(3): 181-187.

[38] Y Q Wu, H Huang, J Zou, et al. Nanoscratch-induced phase transformation of monocrystalline Si. Scripta Materialia, 2010, 63(8): 847-850.

[39] K L Johnson. Contact mechanics. Cambridge: Cambridge University Press, 1985.

[40] L Qian, M Li, Z Zhou, et al. Comparison of nano-indentation hardness and micro hardness. Surface \& Coatings Technology, 2005, 195(2): 264-271.

[41] F Ebrahimi, L Kalwani. Fracture anisotropy in silicon single crystal. Materials Science and Engineering A-structural Materials Properties Microstructure and Processing, 1999, 268(1): 116-126.

[42] B Bhushan. Nano to microscale wear and mechanical characterization using scanning probe microscopy. Wear, 2001, 251(1): 1105-1123.

[43] B Yu, L Qian. Effect of crystal plane orientation on the friction-induced nanofabrication on monocrystalline silicon. Nanoscale Research Letters, 2013, 8(1): 137.

[44] P J Hesketh, C Ju, S Gowda, et al. Surface free energy model of silicon anisotropic etching. Journal of the Electrochemical Society, 1993, 140(4): 1080-1085

[45] P Stempflé, J Takadoum. Multi-asperity nanotribological behavior of single-crystal silicon: crystallography-induced anisotropy in friction and wear. Tribology International, 2012, 48: 35-43.

[46] M C Elwenspoek. On the mechanism of anisotropic etching of silicon. Journal of the Electrochemical Society, 1993, 140(7): 2075-2080.

[47] B Yu. Study on the formation, mechanism and application of friction-induced hillock on monocrystalline silicon. Chengdu: Southwest Jiaotong University, 2012. (in Chinese)

[48] BYu, X Li, H Dong, et al. Mechanical performance of friction-induced protrusive nanostructures on monocrystalline silicon and quartz. Micro \& Nano Letters, 2012, 7(12): 1270-1273.

[49] N Satyanarayana, S K Sinha. Tribology of PFPE overcoated self-assembled monolayers deposited on Si surface. Journal of Physics D: Applied Physics, 2005, 38: 3512-3522.

[50] R Kaneko, S Umemura, M Hirana, et al. Recent progress in microtribology. Wear, 1996, 200(1): 296-304.
[51] S Miyake, J Kim. Fabrication of silicon utilizing mechanochemical local oxidation by diamond tip sliding. Japanese Journal of Applied Physics, 2001, 40(11): 1247-1249.

[52] S Miyake, J Kim. Nanoprocessing of silicon by mechanochemical reaction using atomic force microscopy and additional potassium hydroxide solution etching. Nanotechnology, 2005, 16(1): 149-157.

[53] N Kawasegi, N Morita, S Yamada, et al. Etch stop of silicon surface induced by tribo-nanolithography. Nanotechnology, 2005, 16(8): 1411-1414.

[54] K Chung, Y Lee, D Kim. Characteristics of fracture during the approach process and wear mechanism of silicon AFM tip. Ultramicroscopy, 2005, 102(2): 161-171.

[55] S Miyake, M Wang, J Kim. Silicon nanofabrication by atomic force microscopy-based mechanical processing. Journal of Nanotechnology, 2014: 1-19.

[56] S W Youn, C G Kang. Effect of nanoscratch conditions on both deformation behavior and wet-etching characeristics of silicon (100) surface. Wear, 2006, 261(3): 328-337.

[57] I Zarudi, W C D Cheong, J Zou, et al. Atomistic structure of monocrystalline silicon in surface nano-modification. Nanotechnology, 2004 15(1): 104-107.

[58] A Rota, E Serpini, G C Gazzadi, et al. AFM-based tribological study of nanopatterned surfaces: the influence of contact area instabilities. Journal of Physics: Condensed Matter, 2016, 28(13): 134008.

[59] J W Park, S S Lee, B S So, et al. Characteristics of mask layer on (100) silicon induced by tribo-nanolithography with diamond tip cantilevers based on AFM. Journal of Materials Processing Technology, 2007, 187: 321-325.

[60] I Sung, D Kim. Nano-scale patterning by mechano-chemical scanning probe lithography. Applied Surface Science, 2005, 239: 209-221.

[61] J W Park, N Kawasegi, N Morita. Tribonanolithography of silicon in aqueous solution based on atomic force microscopy. Applied Physics Letters, 2004, 85:1766-1768.

[62] N Kawasegi, N Morita. High-aspect-ratio structure fabrication on (110)-oriented silicon surfaces using tribo-nanolithography. Journal of Nanoscience and Nanotechnology, 2010, 10: 2394-2400.

[63] J W Park, N Kawasegi, N Morita, et al. Mechanical approach to nanomachining of silicon using oxide characteristics based on tribo nanolithography (TNL) in $\mathrm{KOH}$ solution. Journal of Manufacturing Science and Engineering, 2004, 126(4): 801-806.

[64] Y Q Wu, H Huang, J Zou, et al. Nanoscratch-induced deformation of single crystal silicon. Journal of Vacuum Science \& Technology B: Microelectronics and Nanometer Structures Processing, Measurement, and Phenomena, 2009, 27(3): 1374-1377.

[65] H Seidel, L Csepregi, A Heuberger, et al. Anisotropic etching of crystalline silicon in alkaline solutions. Journal of the Electrochemical Society, 1990, 137(11): 3612-3626

[66] DK Biegelsen, M Stutzmann. Hyperfine studies of dangling bonds in amorphous silicon. Physical Review B, 1986, 33(5): 3006-3011.

[67] C Zhou, J Li, L Wu, et al. Friction-induced selective etching on silicon by TMAH solution. RSC advances, 2018, 8(63): 36043-36048.

[68] CY Chen, C P Wong. Unveiling the shape-diversified silicon nanowires made by $\mathrm{HF} / \mathrm{HNO}_{3}$, isotropic etching with the assistance of silver. Nanoscale, 2015, 7(3):1216-1223.

[69] H Wang, C Deng, C Xiao, et al. Fast and maskless nanofabrication for high-quality nanochannel. Sensors and Actuators B: Chemical, 2019, 288: 383-391.

[70] L Wu, B Yu, P Zhang, et al. Rapid identification of ultrathin amorphous damage on monocrystalline silicon surface. Physical Chemistry Chemical Physics, 2020, 22: 12987-12995.

[71] C Jin, B Yu, C Xiao, et al. Temperature-dependent nanofabrication on silicon by friction-induced selective etching. Nanoscale Research Letters, 2016, 11(1): 229.

[72] C Jin, B Yu, X Liu, et al. Site-controlled fabrication of silicon nanotips by indentation-induced selective etching. Applied Surface Science, 2017, 425: 227-232

[73] L Wu, B Yu, Z Fan, et al. Effects of normal load and etching time on current evolution of scratched GaAs surface during selective etching. Materials Science in Semiconductor Processing, 2020, 105: 104744 
[74] J Guo, C Xiao, B Peng, et al. Tribochemistry-induced direct fabrication of nondestructive nanochannels on silicon surface. RSC Advances, 2015 5(122): 100769-100774.

[75] J Guo, B Yu, L Chen, et al. Nondestructive nanofabrication on Si (100) surface by tribochemistry-induced selective etching. Scientific Reports, 2015, 5: 16472.

[76] HWang, B Yu, S Jiang, et al. UV/ozone-assisted tribochemistry-induced nanofabrication on Si (100) surfaces. RSC Advances, 2017, 7(63): 39651-39656.

[77] E Yilgor, O Kaymakci, M Isik, et al. Effect of UV/ozone irradiation on the surface properties of electrospun webs and films prepared from polydimethylsiloxane-urea copolymers. Applied Surface Science, 2012, 258(10): 4246-4253.

[78] X Liu, B Yu, Y Zou, et al. Revealing silicon crystal defects by conductive atomic force microscope. Applied Physics Letters, 2018, 113: 101601.

[79] J Guo, B Yu, X Wang, et al. Nanofabrication on monocrystalline silicon through friction-induced selective etching of $\mathrm{Si}_{3} \mathrm{~N}_{4}$ mask. Nanoscale Research Letters, 2014, 9(1): 241.

[80] C Song, X Li, S Cui, et al. Maskless and low-destructive nanofabrication on quartz by friction-induced selective etching. Nanoscale Research Letters, 2013, 8(1): 140.

[81] C Song, B Yu, L Qian. Effect of scan parameters and etching temperature on low-destructive nanofabrication of quartz. Micro \& Nano Letters, 2013, 8(10): 735-739.

[82] P Tang, B Yu, J Guo, et al. Maskless micro/nanofabrication on GaAs surface by friction-induced selective etching. Nanoscale Research Letters, 2014, 9(1): 59.

[83] P S Pizani, F Lanciotti, R G Jasinevicius, et al. Raman characterization of structural disorder and residual strains in micromachined GaAs. Journal of Applied Physics, 2000, 87(3): 1280.

[84] C Song, B Yu, M Wang, et al. Rapid and maskless nanopatterning of aluminosilicate glass surface via friction-induced selective etching in $\mathrm{HF}$ solution. RSC Advances, 2015, 5(97): 79964-79968.

[85] Y F Niu, K Han, J P Guin. Locally enhanced dissolution rate as a probe for nanocontact-induced densification in oxide glasses. Langmuir, 2012, 28(29): 10733-10740.

[86] C Iliescu, F E H Tay, J Miao. Strategies in deep wet etching of Pyrex glass. Sensors \& Actuators A Physical, 2007, 133(2): 395-400.

[87] L Wu, Z Fan, Y Peng, et al. Rapid nanofabrication via UV-assisted selective etching on GaAs without templates. Chemical Physics Letters, 2019, 717: 152-157.
[88] JV D Ven, H J P Nabben. Analysis of determining factors in the kinetics of anisotropic photoetching of GaAs. Journal of Applied Physics, 1990, 67: 7572-7575.

[89] Y Peng, S Jiang, Y Tan, et al. The formation mechanism of nondefective silicon micropatterns fabricated by scratching assisted electrochemical Etching. ECS Journal of Solid State Science and Technology, 2019, 8(9): 464-471.

[90] V Lehmann, S Ronnebeck. The physics of macropore formation in lowdoped p-type silicon. Journal of the Electrochemical Society, 1999, 146(8): 2968-2975.

[91] V Lehmann, H Foll. Formation mechanism and properties of electrochemically etched trenches in n-type silicon. Journal of the Electrochemical Society, 1990, 137(2): 653-659.

[92] Q Tang, H Shen, H Yao, et al. Formation mechanism of inverted pyramid from sub-micro to micro scale on c-Si surface by metal assisted chemical etching temperature. Applied Surface Science, 2018, 455: 283-294.

[93] L Wu, P Zhang, C Feng, et al. Scanning probe-based nanolithography: nondestructive structures fabricated on silicon surface via distinctive anisotropic etching in $\mathrm{HF} / \mathrm{HNO}_{3}$ mixtures. Journal of Materials Science, 2021, 56: 3887-3899.

[94] ZWu, C Song, J Guo, et al. A multi-probe micro-fabrication apparatus based on the friction-induced fabrication method. Frontiers of Mechanical Engineering, 2013, 8(4): 333-339.

[95] J Chen, J Liu, B Yu, et al. Preparation of multi-tip arrays on flexible substrates for large-scale microfabrication. Micro \& Nano Letters, 2017, 12(2): 104-108.

[96] HWang, B Yu, S Jiang, et al. Simple and low-cost nanofabrication process of nanoimprint templates for high-quality master gratings: Frictioninduced selective etching. Applied Surface Science, 2018, 454: 23-29.

[97] Y Peng, S Jiang, L Xia, et al. Direct ink writing combined with metalassisted chemical etching of microchannels for the microfluidic system applications. Sensors and Actuators A: Physical, 2020, 315: 112320.

[98] R B Schoch, J Han, P Renaud. Transport phenomena in nanofluidics. Reviews of Modern Physics, 2008, 80(3): 839-883.

[99] C Chang, A Sakdinawat. Ultra-high aspect ratio high-resolution nanofabrication for hard X-ray diffractive optics. Nature Communications, 2014, 5(1): $1-7$.

\section{Submit your manuscript to a SpringerOpen ${ }^{\circ}$ journal and benefit from:}

- Convenient online submission

- Rigorous peer review

- Open access: articles freely available online

- High visibility within the field

- Retaining the copyright to your article

Submit your next manuscript at springeropen.com 